Research Paper

\title{
RUNX2 and LAMC2: promising pancreatic cancer biomarkers identified by an integrative data mining of pancreatic adenocarcinoma tissues
}

\author{
Guihua Jin¹, Qingqing Ruan¹, Fugen Shangguan¹, Linhua Lan ${ }^{1}$ \\ ${ }^{1}$ Key Laboratory of Diagnosis and Treatment of Severe Hepato-Pancreatic Diseases of Zhejiang Province, The First \\ Affiliated Hospital of Wenzhou Medical University, Wenzhou 325000, China
}

Correspondence to: Linhua Lan; email: paullee90@163.com, https://orcid.org/0000-0002-6482-1508

Keywords: pancreatic carcinoma, bioinformatics analysis, biomarkers, LAMC2, RUNX2

Received: July 30, $2020 \quad$ Accepted: September 18, $2021 \quad$ Published: October 4, 2021

Copyright: (C) 2021 Jin et al. This is an open access article distributed under the terms of the Creative Commons Attribution License (CC BY 3.0), which permits unrestricted use, distribution, and reproduction in any medium, provided the original author and source are credited.

\begin{abstract}
Pancreatic carcinoma (PC) is a severe disease associated with high mortality. Although strategies for cancer therapy have made great progress, outcomes of pancreatic carcinoma patients remain extremely poor. Therefore, it is urgent to find novel biomarkers and therapeutic targets. To identify biomarkers for early diagnosis and therapy, three mRNA microarray datasets and two miRNA datasets were selected, and combinative analysis was performed by GEO2R. Functional and pathway enrichment analysis were performed using DAVID database. MiRTarBase, miRWalk and Diana Tools were used to get key genes. TCGA, HPA and western blotting were used to verify diagnostic and prognostic value of key genes. By integrating mRNA and miRNA expression profiles, we identified 114 differentially expressed genes and 114 differentially expressed miRNAs, respectively. Then, three overlapping key genes, RUNX2, LAMC2 and FBX032, were found. Their protein levels in pancreatic tissue from PC patients and normal people were analyzed by immunohistochemical staining and western blotting. RUNX2 showed a potential property to identify PC. Aberrant over-expression of LAMC2 was associated with poor prognosis of PC patients, tumor status and subtypes. In summary, our current study identified that RUNX2 and LAMC2 may be promising targets for early diagnosis and therapy of PC patients.
\end{abstract}

\section{INTRODUCTION}

Pancreatic carcinoma (PC) is one of the most malignant types of cancer in the world. Although considerable advancements in diagnostic and pharmacological therapy have been achieved, the 5-year overall survival (OS) rate of PC is still less than 9\% [1]. Thus, novel diagnostic and prognostic biomarkers are urgently needed to improve individualized survival.

Microarray is a high throughput technique used to analyze genetic changes. It has revolutionized studies on RNA and DNA and has also been extensively implemented as an efficient tool for the exploration of genes and biological processes in human diseases. Several studies have reported the differentially expressed genes (DEGs) and differentially expressed microRNA (DEMs) of PC in recent years [2-4]. However, there remain unanswered questions about the interaction between DEGs and DEMs during the progression of PC. And it's necessary for uncovering the real target genes.

In this work, we applied three mRNA profiling datasets (GSE62165 [5], GSE15471 [6] and GSE32676 [7]) and two microRNA (miRNA) profiling datasets (GSE24279 [8] and GSE32678 [7]), which were downloaded from the Gene Expression Omnibus (GEO) database, to obtain DEGs and DEMs between PC and normal tissue samples. Subsequently, DEMs targeted genes (DEMTGs) were predicted, and by overlapping analysis between DEMTGs and DEGs to filter potential genes and microRNAs (miRNAs). We further clarified 
differential expression of key genes through The Cancer Genome Atlas (TCGA) database. Finally, the diagnostic and prognostic values of these key genes and miRNAs were evaluated and predicted by receiver operating characteristic (ROC) and survival analysis.

\section{MATERIALS AND METHODS}

\section{Data collection and DEGs/DEMs/tDEGs/tDEMs analysis}

Gene expression profiles were downloaded from the GEO public database (http://www.ncbi.nlm.nih.gov/ geo/) and the inclusion criteria were as follows: (1) pancreatic cancer, (2) January 1, 2009 to December 31, 2019, (3) homo sapiens, (4) tissue, (5) sample count exceeds 30 , (6) mRNA or miRNA expression data available. As a result, a total of 5 datasets were selected, which included GSE62165, GSE15471, GSE24279, GSE32676 and GSE32678.

The GEO2R web tool was used to screen for DEGs and DEMs between the PC and normal tissue samples in each dataset. The $\log 2$ FoldChange $(\log F C)$ was calculated and the P-values were adjusted to correct for the occurrence of false-positive results by using the default method. Then, P-value $<0.05$ and $|\log 2 \mathrm{FC}|>1.5$ as the cut-off criteria for significant DEGs, and P-value $<0.05$ and $|\log 2 \mathrm{FC}|>0.5$ for significant DEMs was settled. Subsequently, volcano plots of DEGs and DEMs were used to quickly identify differences and Venn analysis was performed to get overlapping up or down regulated DEGs/DEMs in all mRNA/miRNA datasets, respectively [9]. By adding common shared up regulated DEGs/DEMs with down regulated DEGs/DEMs, obtained datasets were named as tDEGs and tDEMs in this study.

\section{Enrichment analysis and key genes acquisition}

Target genes of tDEMs (tDEMTGs) were predicted by the datasets which downloaded from miRTarBase (http:// miRTarBase.cuhk.edu.cn/), miRWalk (http://mirwalk. umm.uni-heidelberg.de/) and Diana Tools (http://diana. imis.athena-innovation.gr/DianaTools/index.php) $\quad[10$ 12]. Gene ontology (GO) and Kyoto Encyclopedia of Genes and Genomes (KEGG) were applied for the potential relevant functional annotation and pathway enrichment analysis of tDEMTGs and tDEGs were analyzed by the Database for Annotation Visualization and Integrated Discovery (DAVID, *https://david. ncifcrf.gov/) [13]. Then, tDEMTGs were aligned with tDEGs to obtain the intersection termed "key genes" for further analysis. Additionally, tDEMs which were relevant to key gene regulation termed as "candidate miRNAs".

\section{Survival analysis}

To evaluate the prognostic value of key genes and miRNAs, the overall survival (OS) of PC patients was investigated based on the online database, KaplanMeier Plotter (http://kmplot.com/analysis/index.php) [14]. High- and low-level cohorts of the indicated genes were set according to the median value of gene expressions. The OS plot was obtained with the hazard ratio (HR), the $95 \%$ confidence interval (CI) and logrank P-value information.

\section{Key gene expression analysis in TCGA and genotype-tissue expression (GTEx) tissues}

Gene Expression Profiling Interactive Analysis (GEPIA [15], http://gepia.cancer-pku.cn/) is an interactive web server for estimating the RNA-Seq expression data from the TCGA (https://www.cancer.gov/about-nci/ organization/ccg/research/structural-genomics/tcga) and GTEx (https://www.gtexportal.org/) databases. The expression of key genes was analyzed in various tumor and non-tumor tissues using GEPIA, and the comparison of the key gene expression at different stages of PC was also performed. The clinical information of patients was also downloaded from TCGA for further data validation.

\section{Data validation}

Blood and saliva GEO data verification: We investigated a validation by comparing the mRNA standardized values of key genes in five independent GEO datasets. Four of them were blood samples (GSE74629 [16], GSE49641 [17], GSE49515 [18] and GSE15932) and the last one was saliva samples (GSE14245 [19]).

Immunohistochemistry (IHC) verification: The IHC staining images of PC and normal tissue, which obtained from the Human Protein Atlas [20] (HPA, https://www.proteinatlas.org), were used as validation of key genes. The staining, intensity, quantity and location of IHC images present in HPA were calculated for each gene. (For the more details about statistical methods of the IHC images, the reader is referred to the web page https://www.proteinatlas.org/about/assays+ annotation\#ih annotation).

Western blotting (WB) verification: Sixteen pancreatic tissue samples from PC patients were obtained by surgical resection and further divided into tumor group and paracancerous group (normal). The study was approved by the Institutional Ethics Committee of Wenzhou Medical University and written informed consent was obtained from each patient before their enrollment. WB protocol was performed according to our previously described procedures [21]. The 
following primary antibodies were used: RUNX2 (ABclonal, cat. no. A2851), LAMC2 (ABclonal, cat. no. A1869), AKT (Cell signaling Technology, cat. no. \#4685), p-AKT (Cell signaling Technology, cat. no. \#4060), p-c-Raf (Cell signaling Technology, cat. no. \#9421), p-MEK1/2 (Cell signaling Technology, cat. no. \#9154), p-ERK1/2 (Cell signaling Technology, cat. no. \#4370), p-p90RSK (Cell signaling Technology, cat. no. \#9346), p-MSK1 (Cell signaling Technology, cat. no. \#9595), and $\beta$-Actin (Abmart, cat. no. P30002). The protein expression levels were quantified by Image $\mathbf{J}$ software.

\section{Statistical analysis}

The discriminative ability of key genes and miRNAs in the GEO datasets was calculated by ROC analysis, the pROC [22] $R$ package was performed in $R$ 3.6.2 (http://www.R-project.org/). The area under the curve (AUC), 95\% CI and P-value for assessing the predictive accuracy and specificity of ROC were calculated by SPSS version 23.0 (IBM, Chicago, IL, USA). All scatter and bar plots were generated by Graph Pad Prism 7 Software (GraphPad Software, Inc.). Comparisons among means were performed by ANOVA. P-value $<0.05$ was assessed as statistically significant.

\section{RESULTS}

\section{Initial identification of tDEGs, tDEMs and tDEMTGs in pancreatic cancer}

To find key genes that were differentially expressed between PC patients and healthy people, we decided to filter them both ways: mRNA and miRNA targeted method. Firstly, we selected three expression array profiling datasets (GSE62165, GSE15471 and GSE32676) and two non-coding RNA array profiling datasets (GSE24279 and GSE32678) from GEO database. As for mRNA, 1398, 607 and 1157 DEGs were extracted from GSE62165, GSE15471 and GSE32676. Among them, there were 997, 562, 669 (103 shared genes) upregulated and 401, 45, 488 (11 shared genes) downregulated DEGs were identified. Taking advantage of Venn analysis in up or down regulated DEGs respectively, we captured 114 total-shared DEGs (tDEGs). Among them, there were 103 up-regulated and 11 down-regulated genes (Figure 1A, 1B). As for miRNA, the same operations were also performed. 417 and 569 DEMs were extracted from GSE24279 and GSE32678. Two miRNA datasets shared 66 total DEMs (tDEMs), including 24 up-regulated and 42 downregulated miRNAs (Figure 1C, 1D).

Target genes for tDEMs were predicted by miRTarBase, miRWalk and Diana Tools databases.
Then 114 consistent genes, which we termed as tDEMTGs, were found by Venn's analysis (Figure 1E).

\section{GO and pathway enrichment analysis}

The GO term and KEGG pathway analysis were performed via DAVID. First, the results of the GO enrichment analysis varied a lot between tDEGs and tDEMTGs (Figure 2A, 2B). Biological process (BP) analysis of GO showed tDEGs were significantly enriched in extracellular matrix associated part, such as cell adhesion, extracellular matrix organization and disassembly, collagen catabolism and organization. As for tDEMTGs, top 2 clusters of genes were enriched in the regulation of transcription and proliferation, which showed a similar result in tDEGs, but other genes were mainly responsible for the regulation of cell death associated part. For GO cell component (CC) analysis, the tDEGs were significantly enriched in extracellular part, such as extracellular region, extracellular exosome, extracellular space, extracellular matrix and proteinaceous extracellular matrix. However, the results of tDEMTGs were focused on plasma membrane part, cytosol, organelle lumen, membrane-enclosed lumen and intracellular organelle lumen. The same differences also showed in molecular function (MF) analysis, tDEGs were mainly enriched in protein binding, but tDEMTGs were enriched in nucleotide binding, transcription regulator activity, ribonucleotide binding, purine ribonucleotide binding and purine nucleotide binding.

Additionally, the differences between tDEGs and tDEMTGs were also confirmed by KEGG pathway analysis. The result disclosed that tDEGs were involved in PI3K/Akt signaling pathway, focal adhesion, ECMreceptor interaction, amoebiasis and pathways in cancer (Figure 2C). However, tDEMTGs were significantly enriched in pathways in cancer and partial enriched in pancreatic cancer (Figure 2D).

\section{Identification of key genes and miRNAs}

To identify potential key genes, tDEMTGs were aligned with tDEGs to get the cross-section, which we termed as key genes for further analysis (Figure 1F). Importantly, the result showed that runt related transcription factor 2 (RUNX2), laminin subunit gamma-2 (LAMC2) and F-box protein 32 (FBXO32) were commonly shared and then listed in Table 1 . Subsequently, we analyzed candidate miRNAs involved in the regulation of key genes from 66 tDEMs, and found 16 up-regulated and 19 down-regulated key miRNAs (Table 2). 


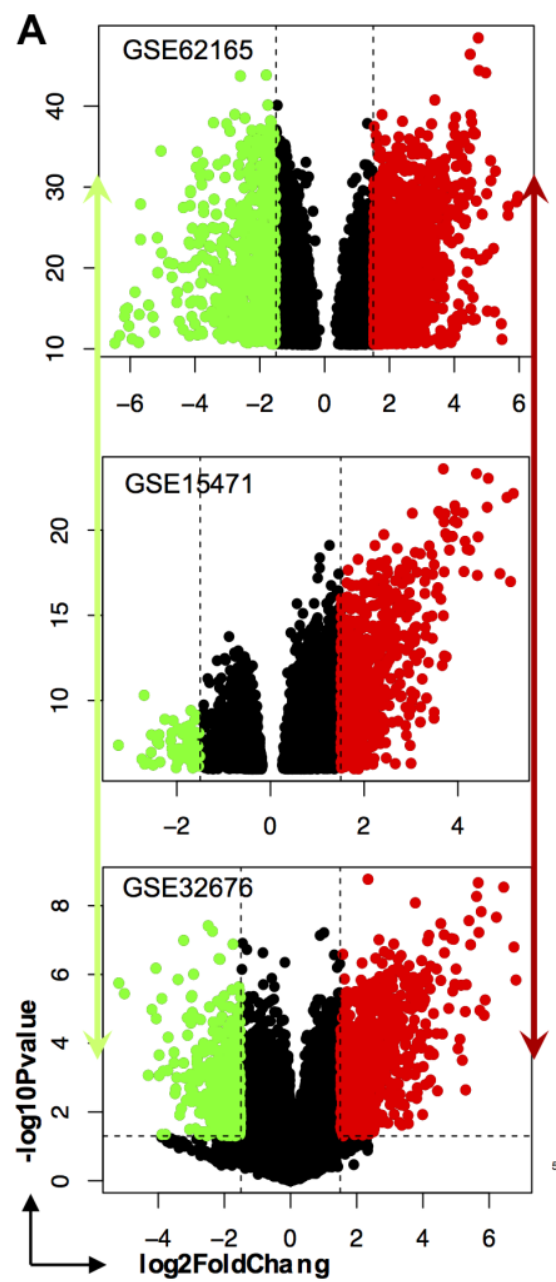

B
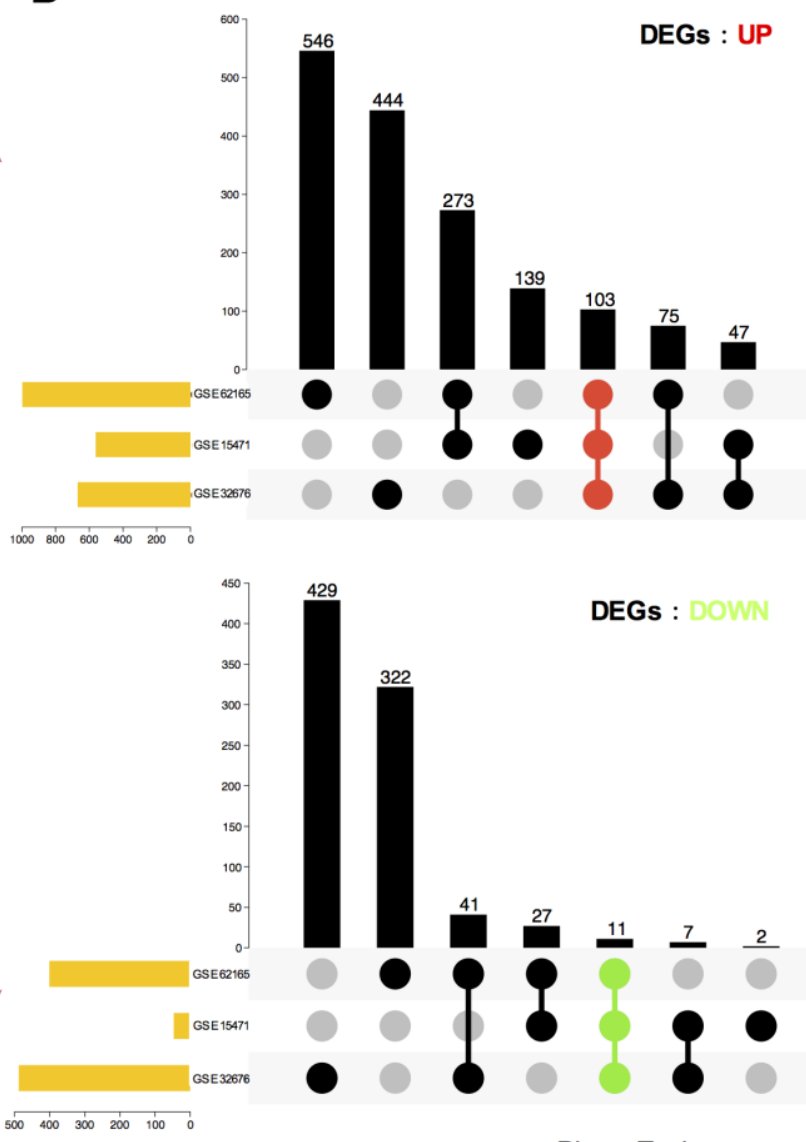

C

D
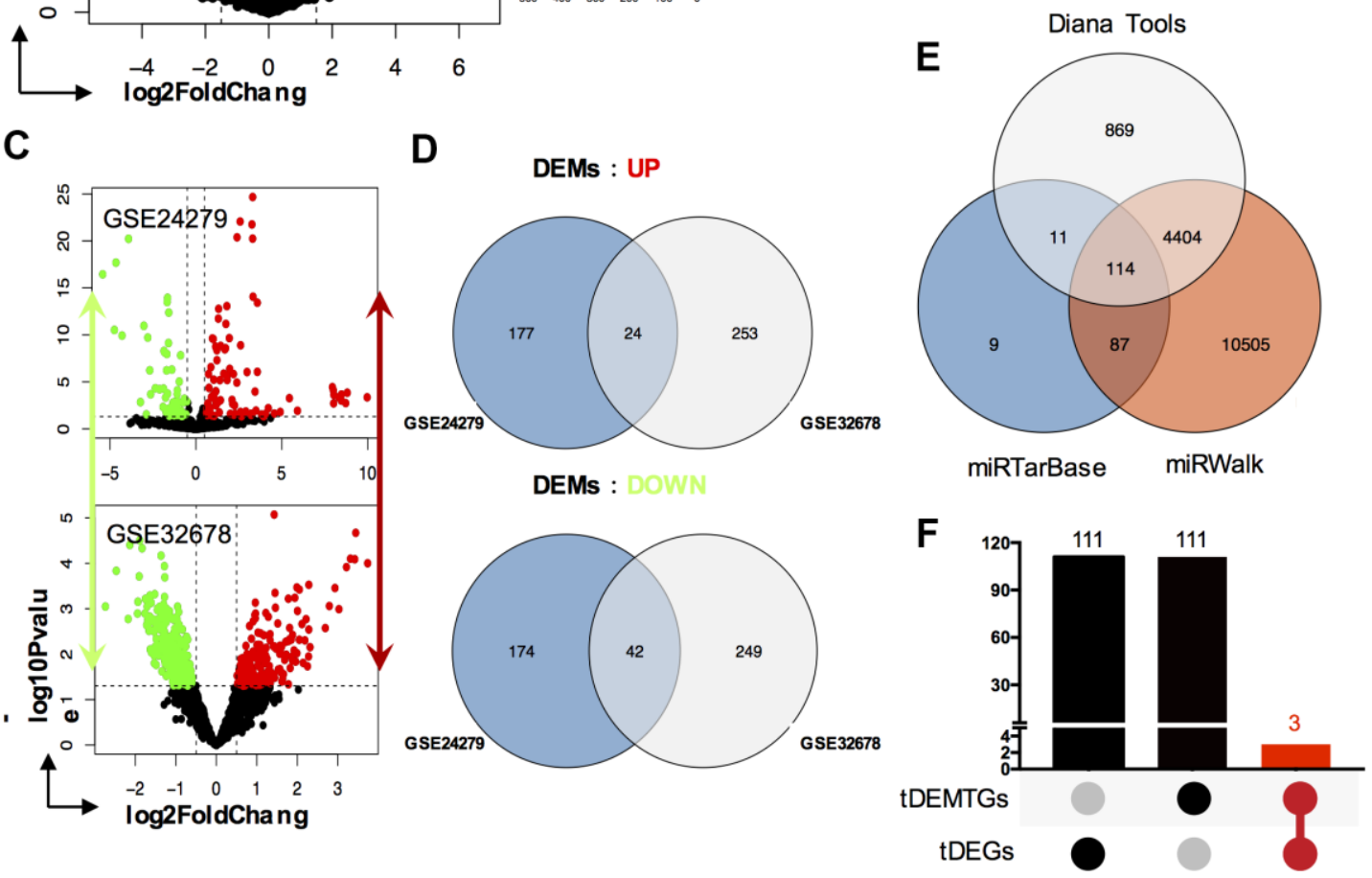

Figure 1. Identification of DEGs, DEMs and key genes. (A) The volcano plot of mRNA GEO datasets GSE62165, GSE15471 and GSE32676 respectively; (B) Venn diagram of up-regulated DEGs and down-regulated DEGs set from GSE62165, GSE15471 and GSE32676 datasets; (C) The volcano plot of miRNA GEO datasets GSE24279 and GSE32678 respectively; (D) Venn diagram of up-regulated DEMs and down-regulated DEMs set from GSE24279 and GSE32678 datasets; (E) The target genes, tDEMTGs, of 66 consistent DEMs (tDEMs) were predicted by miRTarBase, miRWalk and Diana Tools databases. (F) Venn diagram of tDEMTGs and tDEGs to get key genes. 
A
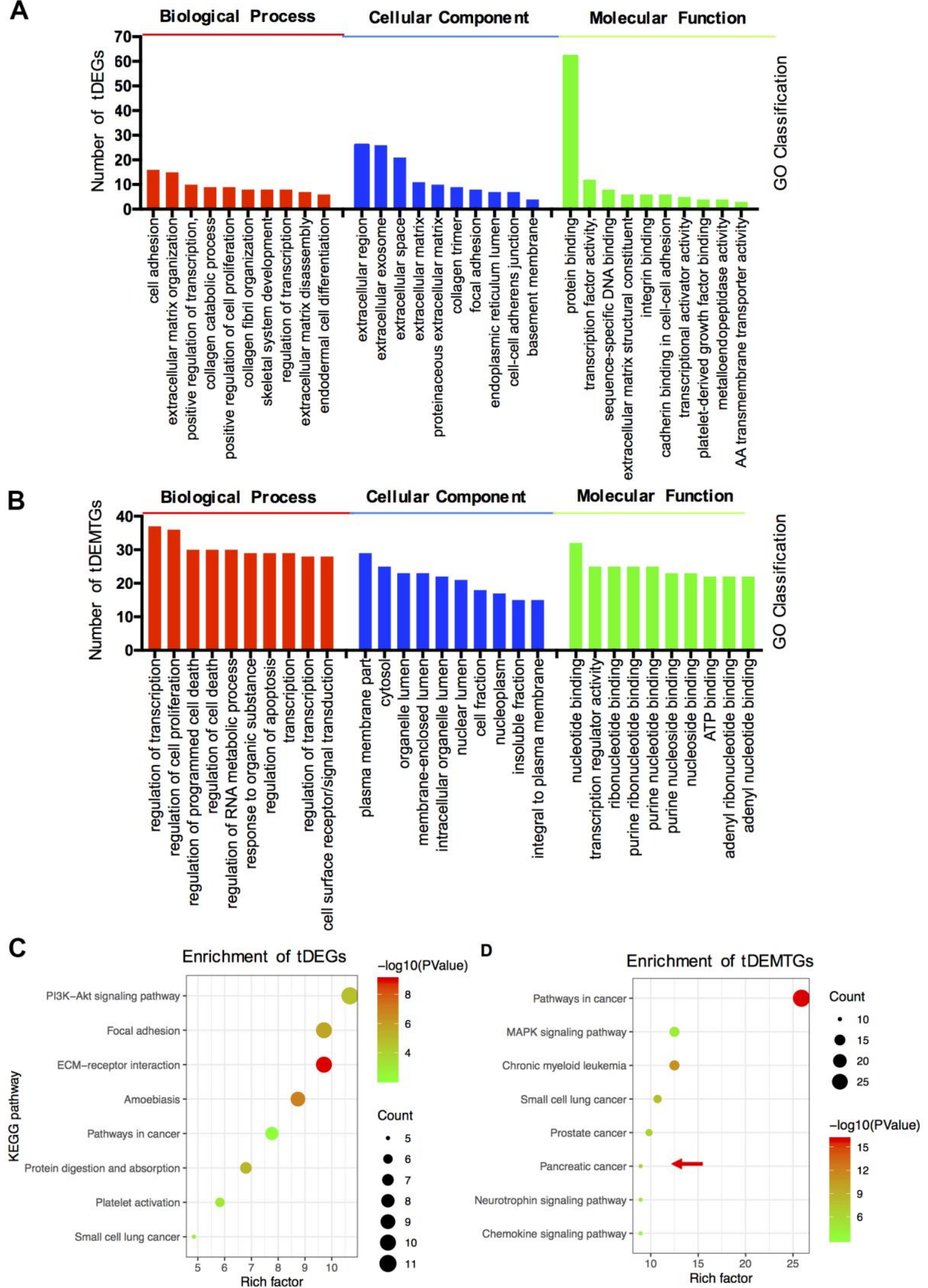

Figure 2. The functional and pathway enrichment analysis of tDEGs and tDEMTGs. (A) Biological process, cellular component and molecular function analysis for tDEGs, top 10 of each GO classification were listed; (B) Biological process, cellular component and molecular function analysis for tDEMTGs; (C) KEGG pathway enrichment analysis for tDEGs, top 8 were listed; (D) KEGG pathway enrichment analysis for tDEMTGs. 
Table 1. The differential expression of key genes in different GEO datasets.

\begin{tabular}{|c|c|c|c|c|c|}
\hline Gene & Datasets & ID & LogFC & P.Val & Expression \\
\hline \multirow{3}{*}{ RUNX2 } & GSE62165 & 11725180_a_at & 3.7 & $3.10 \mathrm{E}-29$ & UP \\
\hline & GSE15471 & 232231_at & 2.93 & $9.52 \mathrm{E}-11$ & UP \\
\hline & GSE32688(mRNA) & 232231_at & 1.69 & 0.1046045 & UP \\
\hline \multirow{9}{*}{ LAMC2 } & \multirow{5}{*}{ GSE62165 } & 11737325_a_at & 3.49 & $4.80 \mathrm{E}-25$ & UP \\
\hline & & 11743514_a_at & 4.09 & $7.56 \mathrm{E}-26$ & UP \\
\hline & & 11743516_s_at & 4.29 & $1.49 \mathrm{E}-26$ & UP \\
\hline & & 11743515_s_at & 4.35 & $1.06 \mathrm{E}-28$ & UP \\
\hline & & 11747923_s_at & 4.37 & $7.93 \mathrm{E}-31$ & UP \\
\hline & \multirow{2}{*}{ GSE15471 } & 207517_at & 1.60 & $3.83 \mathrm{E}-09$ & UP \\
\hline & & 202267_at & 2.76 & $1.76 \mathrm{E}-13$ & UP \\
\hline & \multirow{2}{*}{ GSE32688(mRNA) } & 202267_at & 4.93 & 0.0002728 & UP \\
\hline & & 207517_at & 2.25 & 0.0038994 & UP \\
\hline \multirow{8}{*}{ FBXO32 } & & 11719394_a_at & 2.88 & $3.83 \mathrm{E}-22$ & UP \\
\hline & GSE62165 & 225803_at & 1.72 & $9.65 \mathrm{E}-14$ & UP \\
\hline & \multirow{3}{*}{ GSE15471 } & 241762_at & 1.83 & 2.64E-11 & UP \\
\hline & & 225328_at & 1.91 & $5.28 \mathrm{E}-12$ & UP \\
\hline & & 241763_s_at & 2.44 & 0.0095169 & UP \\
\hline & \multirow{3}{*}{ GSE32688(mRNA) } & 241762_at & 2.32 & 0.0215962 & UP \\
\hline & & 225803_at & 1.88 & 0.0176426 & UP \\
\hline & & 225345_s_at & 1.56 & 0.0702839 & UP \\
\hline
\end{tabular}

Table 2. The differential expression of candidate miRNAs in different GEO datasets.

\begin{tabular}{|c|c|c|c|c|}
\hline Target gene & miRNA & Datasets & $\log \mathrm{FC}$ & Expression \\
\hline \multirow{14}{*}{ RUNX2 } & \multirow{2}{*}{$\underline{\text { hsa-miR-519c-3p }}$} & GSE32678 & 0.531577 & \multirow[t]{2}{*}{ UP } \\
\hline & & GSE24279 & 3.2867924 & \\
\hline & \multirow{2}{*}{ hsa-miR-876-3p } & GSE32678 & 0.755198 & \multirow[t]{2}{*}{ UP } \\
\hline & & GSE24279 & 9.9730133 & \\
\hline & \multirow{2}{*}{$\underline{\text { hsa-miR-1265 }}$} & GSE32678 & 0.883218 & \multirow[t]{2}{*}{ UP } \\
\hline & & GSE24279 & 0.6795887 & \\
\hline & \multirow{2}{*}{ hsa-miR-92a-2-5p } & GSE32678 & -1.231335 & \multirow[t]{2}{*}{ DOWN } \\
\hline & & GSE24279 & -0.7331099 & \\
\hline & \multirow{2}{*}{ hsa-miR-193b-5p } & GSE32678 & 0.6020578 & \multirow[t]{2}{*}{ DOWN } \\
\hline & & GSE24279 & -1.218209 & \\
\hline & \multirow{2}{*}{ hsa-miR-517-5p } & GSE32678 & -0.606971 & \multirow[t]{2}{*}{ DOWN } \\
\hline & & GSE24279 & -1.2242733 & \\
\hline & \multirow{2}{*}{ hsa-miR-488-3p } & GSE32678 & -1.145003 & \multirow[t]{2}{*}{ DOWN } \\
\hline & & GSE24279 & -1.145003 & \\
\hline \multirow{8}{*}{ RUNX2/FBXO32 } & \multirow{2}{*}{ hsa-miR-23b-3p } & GSE32678 & 1.214636 & \multirow[t]{2}{*}{ UP } \\
\hline & & GSE24279 & 0.7358448 & \\
\hline & \multirow{2}{*}{ hsa-miR-221-5p } & GSE32678 & 1.277623 & \multirow[t]{2}{*}{ UP } \\
\hline & & GSE24279 & 1.8034373 & \\
\hline & \multirow{2}{*}{ hsa-miR-199a-5p } & GSE32678 & 1.240204 & \multirow[t]{2}{*}{ UP } \\
\hline & & GSE24279 & 1.6889355 & \\
\hline & \multirow{2}{*}{ hsa-miR-376a-5p } & GSE32678 & 0.546421 & \multirow[t]{2}{*}{ UP } \\
\hline & & GSE24279 & 2.3186641 & \\
\hline
\end{tabular}




\begin{tabular}{|c|c|c|c|c|}
\hline & bco mid 1825 & GSE32678 & 1.225039 & UP \\
\hline & $\underline{\text { hsa-mik-1825 }}$ & GSE24279 & 4.0684489 & \\
\hline & he-miR $1227-3 n$ & GSE32678 & 0.687478 & UP \\
\hline & hsa-mik-122/-3p & GSE24279 & 3.0619077 & \\
\hline & hes-miR_198 & GSE32678 & -1.004687 & DOWN \\
\hline & hsa-mik-198 & GSE24279 & -0.6024842 & \\
\hline & b & GSE32678 & -0.768939 & DOWN \\
\hline & nsa-mik-93 $-5 \mathrm{p}$ & GSE24279 & -1.2399531 & \\
\hline FBXO32 & & GSE32678 & 0.821379 & UP \\
\hline & hsa-miR-345-5p & GSE24279 & 7.9474776 & \\
\hline & & GSE32678 & 0.528843 & UP \\
\hline & hsa-miR-1233-3p & GSE24279 & 1.0034567 & \\
\hline & & GSE32678 & 0.583983 & UP \\
\hline & hsa-miR-1289 & GSE24279 & 0.5373088 & \\
\hline & bo mid 601 & GSE32678 & 0.962951 & UP \\
\hline & nsa-mik-021 & GSE24279 & 0.9769538 & \\
\hline & b & GSE32678 & 1.377747 & UP \\
\hline & $\underline{n s a-m i k-1248}$ & GSE24279 & 1.0123159 & \\
\hline & hca-miR-30b-3n & GSE32678 & -0.705179 & DOWN \\
\hline & 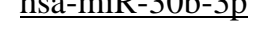 & GSE24279 & -0.7012921 & \\
\hline & hea-miR-373-5n & GSE32678 & -0.943119 & DOWN \\
\hline & 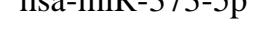 & GSE24279 & -1.5962925 & \\
\hline & hea-miR_49? & GSE32678 & -0.665303 & DOWN \\
\hline & nsa-mik-492 & GSE24279 & -1.1011978 & \\
\hline & 5 & GSE32678 & -1.069006 & DOWN \\
\hline & nsa-mIK-J10-गp & GSE24279 & -2.499187 & \\
\hline & bo mid 575 & GSE32678 & -0.77259 & DOWN \\
\hline & חSA-ImIK-J/S & GSE24279 & -0.5620953 & \\
\hline & be mip 604 & GSE32678 & -0.508662 & DOWN \\
\hline & 115a-mIK-out & GSE24279 & -1.2474484 & \\
\hline & bor mid 611 & GSE32678 & -1.159661 & DOWN \\
\hline & 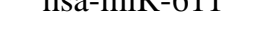 & GSE24279 & -1.6342346 & \\
\hline & bo miD 617 & GSE32678 & -0.816815 & DOWN \\
\hline & hsa-mik-61/ & GSE24279 & -1.5017728 & \\
\hline & be mid 021 & GSE32678 & -0.90506 & DOWN \\
\hline & 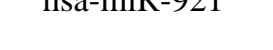 & GSE24279 & -1.9104782 & \\
\hline & hea-miR-520d-5n & GSE32678 & -0.660262 & DOWN \\
\hline & & GSE24279 & -0.6523876 & \\
\hline ГРОУ2ר/ А & b & GSE32678 & 1.544751 & UP \\
\hline FBUX 32/LAMCZ & nsa-mik-199b-5p & GSE24279 & 1.0106128 & \\
\hline & & GSE32678 & 0.526075 & UP \\
\hline & hsa-miR-588 & GSE24279 & 4.3557666 & \\
\hline LAMC? & & GSE32678 & -0.713461 & DOWN \\
\hline LAMC2 & hsa-miR-89la-5p & GSE24279 & -1.3790634 & \\
\hline & & GSE32678 & -0.961764 & DOWN \\
\hline & hsa-miR-622 & GSE24279 & -1.1362947 & \\
\hline LAMC2/RUNX2 & hsa-miR-628-3p & GSE32678 & -0.64033 & DOWN \\
\hline & $1 \mathrm{ISa}-1 \mathrm{MIR}-0 \angle \mathrm{O}^{-3 p}$ & GSE24279 & -0.9205919 & \\
\hline
\end{tabular}




\section{Diagnostic and prognostic values of candidate miRNAs}

To test the clinical applicability of the candidate miRNAs, we investigated their diagnostic significance through ROC curve analysis (Supplementary Figure 1A). However, the mean AUC values of them were all lower than 0.85 (Supplementary Figure 1B). To further evaluate their prognostic value, survival analysis was applied and the results showed 10 up-regulated key miRNAs indicated a significantly lower OS rate: hsamiR-519c-3p, hsa-miR-1265, hsa-miR-1825, hsa-miR1227-3p, hsa-miR-1233-3p, hsa-miR-1289, hsa-miR621, hsa-miR-1248, hsa-miR-199b-5p and hsa-miR-588 (Figure 3A). Correspondingly, 3 down-regulated candidate miRNAs were positively related with OS rate: hsa-miR-488-3p, hsa-miR-30b-3p and hsa-miR-628-3p (Figure 3B). These data indicated that these 13 candidate miRNAs could be potential prognostic markers for clinical application.

\section{Diagnostic and prognostic values of key genes}

We then compared the transcriptional levels of key genes in several cancers with those of normal samples using TCGA database via GEPIA (Figure 4A-4C). The mRNA expression levels of RUNX2, LAMC2 and FBXO32 showed significant differences between cancer and adjacent non-cancerous tissues especially in pancreatic adenocarcinoma (PAAD, Figure 4D). ROC and survival analysis were also performed to ensure the application prospect of key genes. All the three key genes indicated a good discriminating ability, of which the mean AUC values of each key gene in three GEO datasets were all greater than 0.85 (Figure 4E). Furthermore, the mean AUC values of LAMC2 and

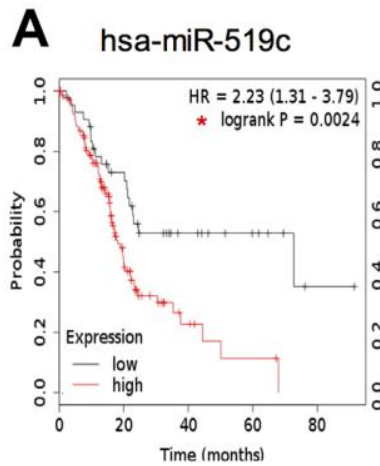

hsa-miR-1265

hsa-miR-1825

hsa-miR-1227

hsa-miR-1248
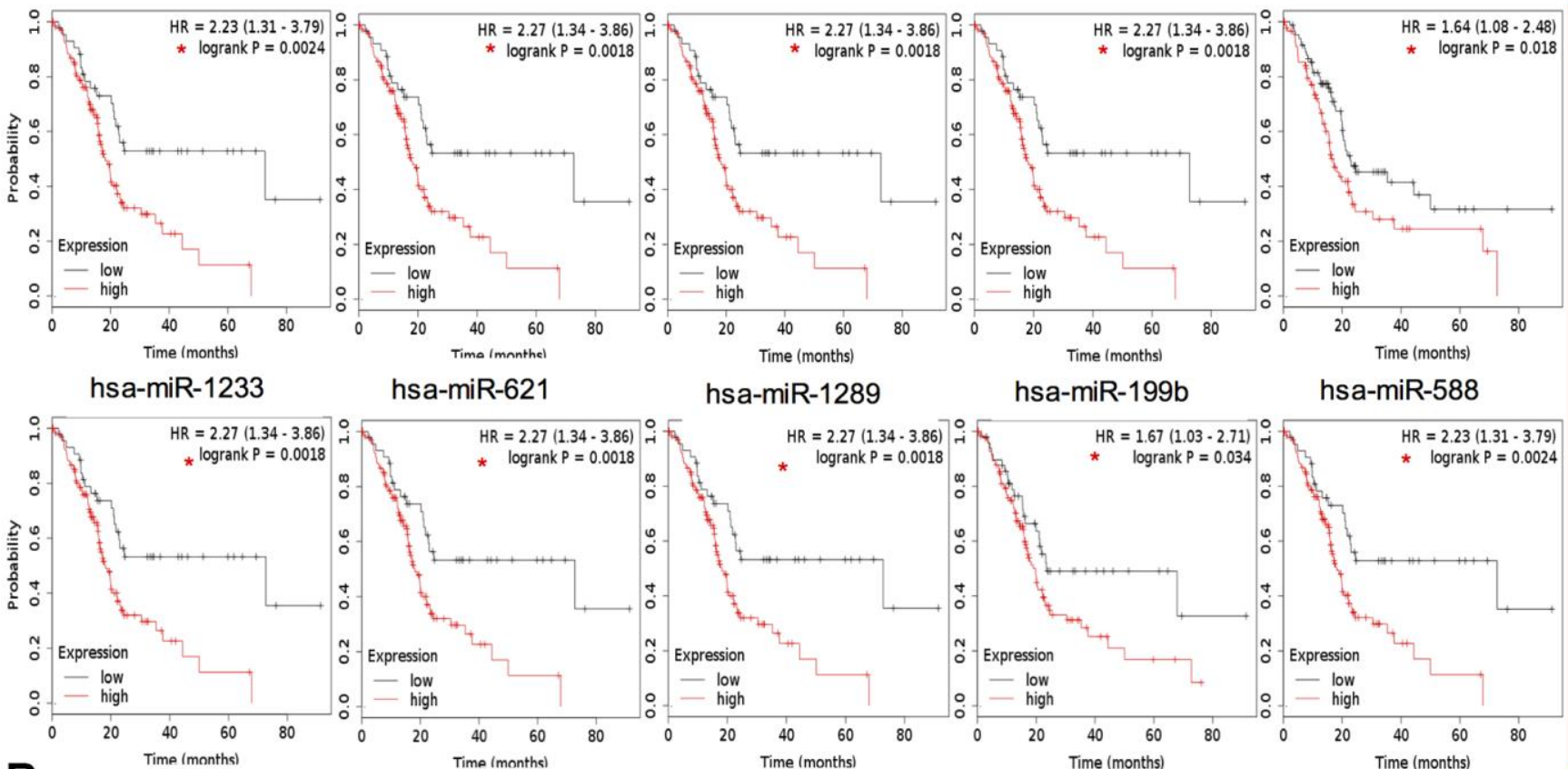

hsa-miR-621

hsa-miR-1289

hsa-miR-199b
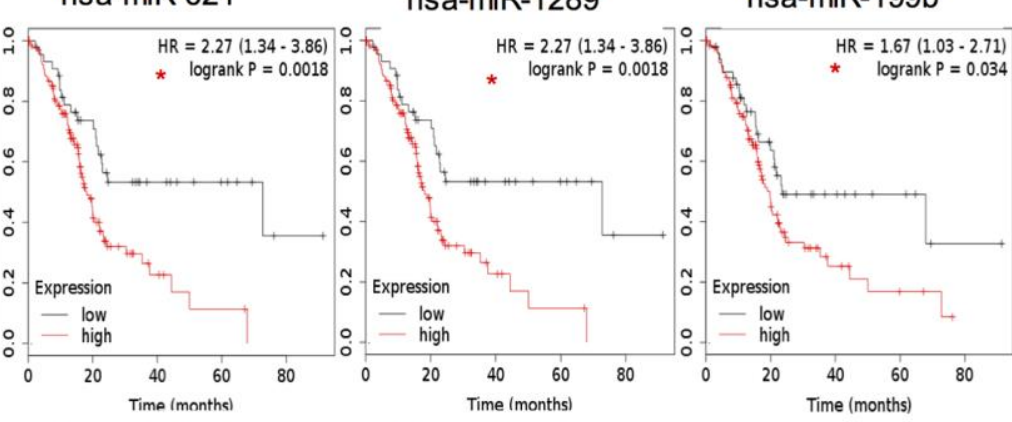

hsa-miR-588

B hsa-miR-30b

hsa-miR-488

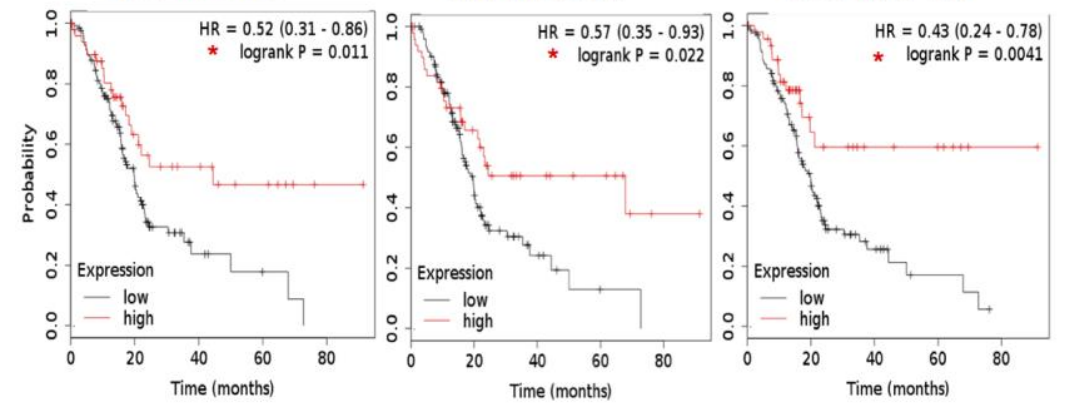

$* P<0.05$

Up-regulated miRNAs

Down-regulated miRNAs

Figure 3. Survival analysis of 13 key DEMs expression in TCGA PAAD cohort. Red color indicates upregulation (A), whereas green color indicates that the miRNA is downregulated (B). 

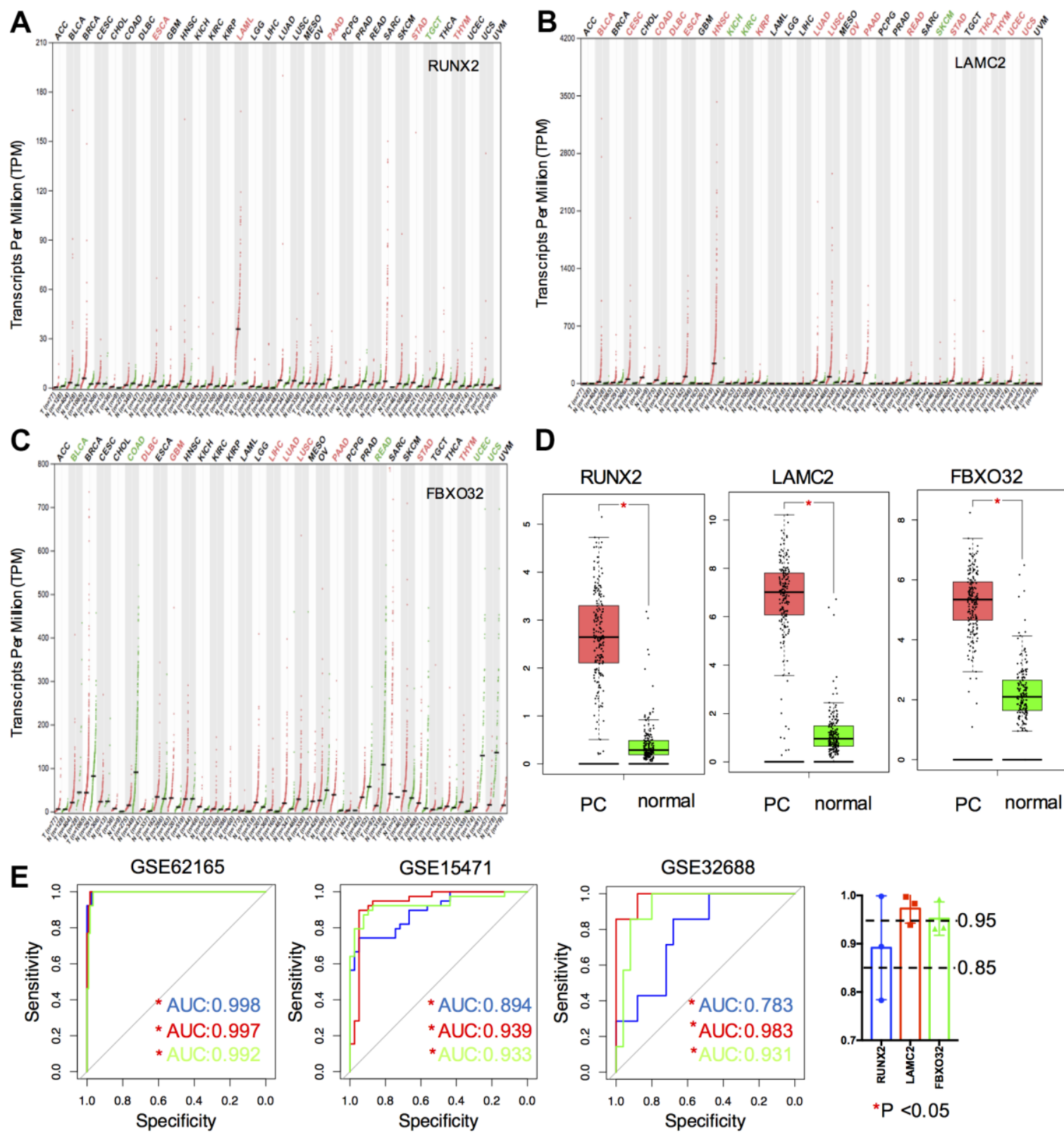

D
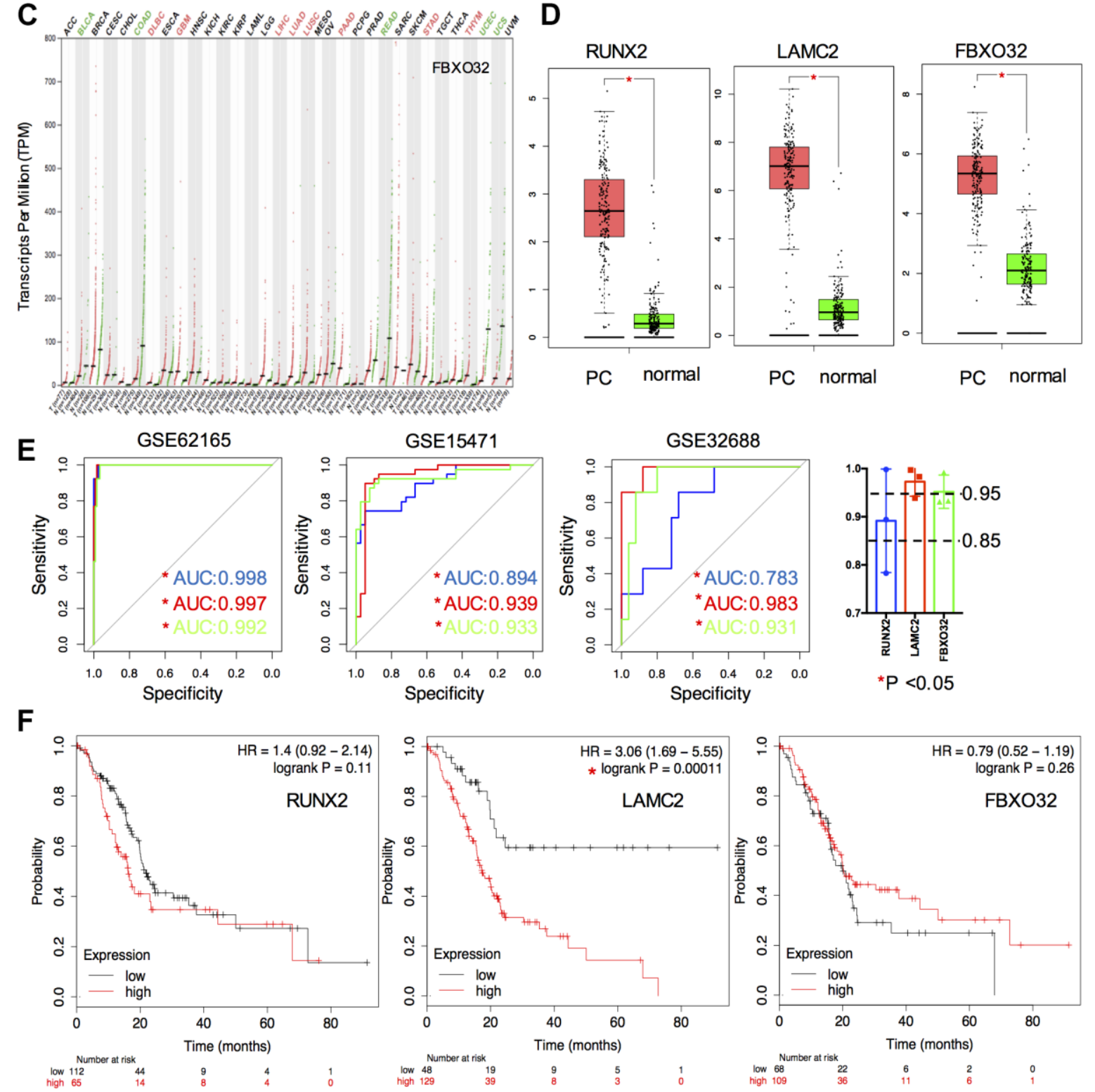

Figure 4. Expression, ROC and survival analysis of key genes. (A-C) Differential expression of key genes (RUNX2, LAMC2 and FXBO32) in various tumor and non-tumor tissues. (D) Differential expression of key genes in PC patients and normal controls. (E) ROC curve of key genes in GSE62165, GSE15471 and GSE32688. (F) Survival analysis of key genes in TCGA PAAD cohort. 
FBXO32 were both greater than 0.95 which indicated that these two molecules might be promising biomarkers for PC diagnosis. The results of survival analysis showed that only the LAMC2 expression level affected $\mathrm{OS}$ rate significantly $(\mathrm{HR}=3.06, \mathrm{P}=0.00011$, Figure 4F). It suggested that LAMC2 could be an important molecule which participate in the development of PC and also act as a potential prognostic biomarker.

\section{Verification of key genes}

In addition to high accuracy and specificity, the diagnostic methods should also be painless and woundless. Blood and saliva are easily obtained specimens that we can get with little body damage. Thus, we verified the diagnostic possibility of key genes in 4 blood GEO datasets (GSE74629, GSE49641, GSE49515 and GSE15932) and 1 saliva GEO dataset (GSE14245). Interestingly, we found only RUNX2 had significant reduction compared with normal controls in saliva and blood cells (GSE14245 and GSE49641), which suggested that RUNX2 could be considered as one of promising candidate biomarkers of PC. LAMC2 and $\mathrm{FBXO} 32$ had no significant differences between the control and PC samples.

Then, to further clarify the expression of RUNX2 and LAMC2 in PC tissues, the results of IHC images of these two genes in HPA were explored. (Figure 5B and Supplementary Figure 2). First, the expression of LAMC2 was consistent with previous analysis. The staining of anti-LAMC2 antibodies were all above medium level in PC patients, which were barely detected in normal pancreas tissues. Although staining quantity were varied $(5.5 / 12$ were $>75 \%, 2.5 / 12$ were $75 \%$ $25 \%$ and $4 / 12$ were $<25 \%$ stained), almost all of their staining intensity were strong. However, the result of RUNX2 was not corroborated with predicted. Though a slight up-regulation of RUNX2 could be found in some samples (17\%), most could not be detected. In addition, our immune blot results were consistent

A
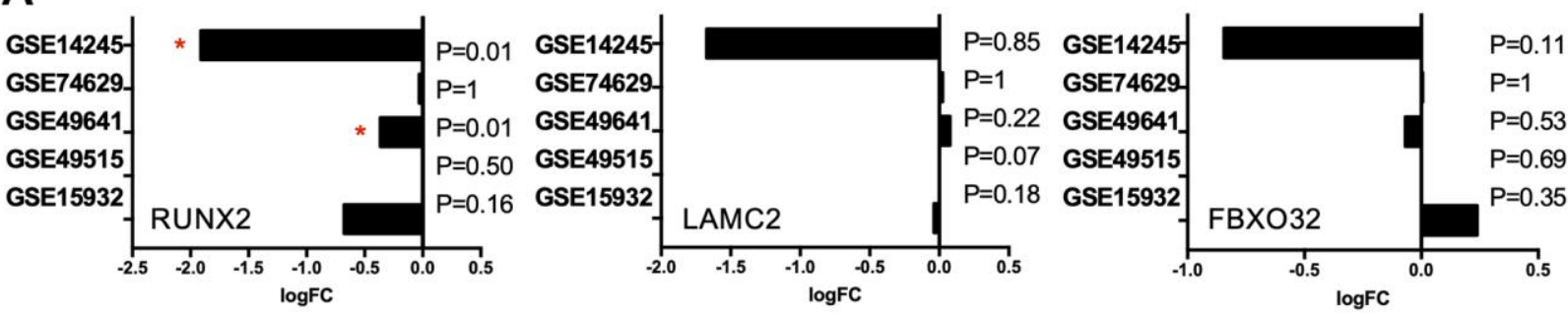

B
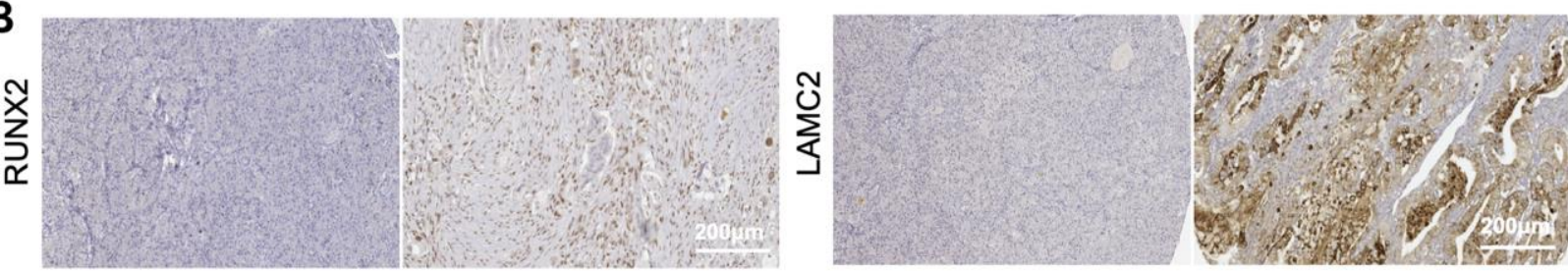

Normal tissue

PC tissue

Normal tissue

$\mathrm{PC}$ tissue

C
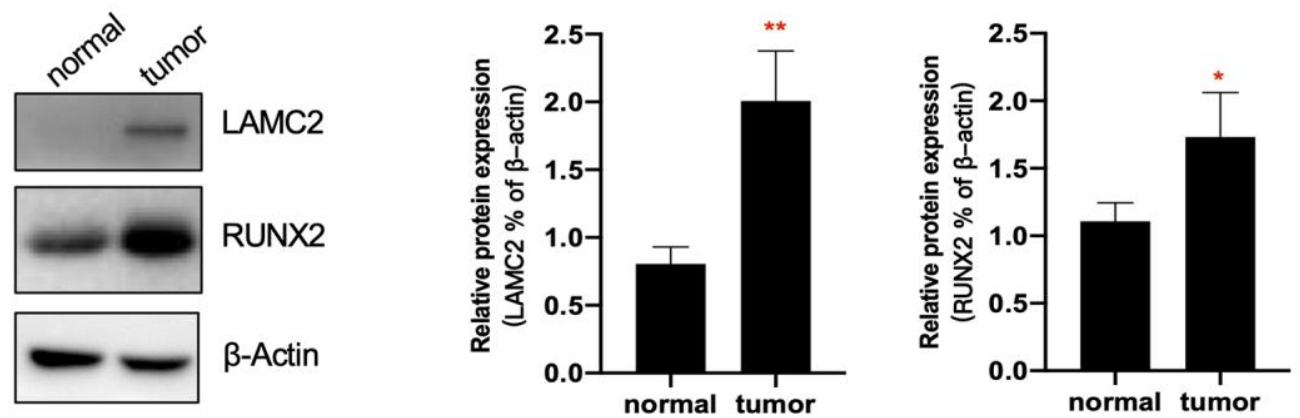

Figure 5. Verification of key genes. (A) RUNX2, LAMC2 and FXBO32 expression levels (Log2FoldChange value) in GSE14245, GSE74629, GSE49641, GSE49515 and GSE15932. (B) Representative immunohistochemistry staining of RUNX2 and LAMC2 in pancreatic ductal adenocarcinoma (PC tissue) and control pancreatic tissue (Normal tissue) in Human Protein Atlas (HPA). Scales bars represent $200 \mu \mathrm{m}$. (C) Western blot analysis for RUNX2 and LAMC2 expression proteins in 16 paired samples from PC patients. 
with IHC experiment (Figure 5C). These data indicated that LAMC2 may play a crucial role in the PC therapy.

In a second validation study, we explored the expression of RUNX2 and LAMC2 in different clinical stage, sex, age, tumor subtype and personal tumor status groups (Figure 6A-6E). We found RUNX2 was only significantly up-regulated in advanced stage groups.
However, LAMC2 were significantly elevated not only in advanced PC patients, but also in the ductal type PC group $(\mathrm{P}=0.03)$ and $\mathrm{PC}$ patients which with tumor group $(\mathrm{P}=0.02)$.

According to all the above evidence, we found LAMC2 could be the major factor influencing the median survival time of PC patients through combinatorial
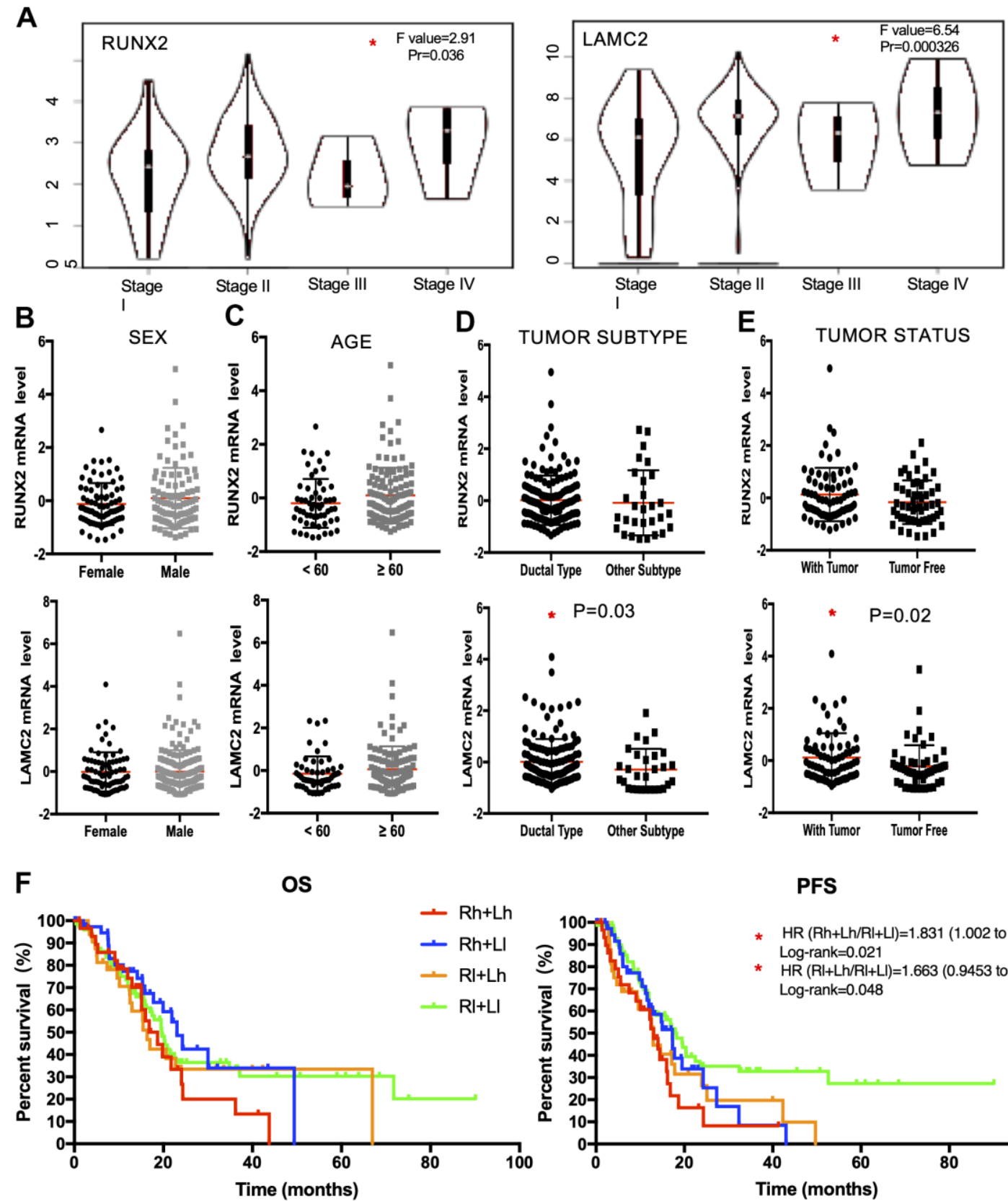

\section{PFS}

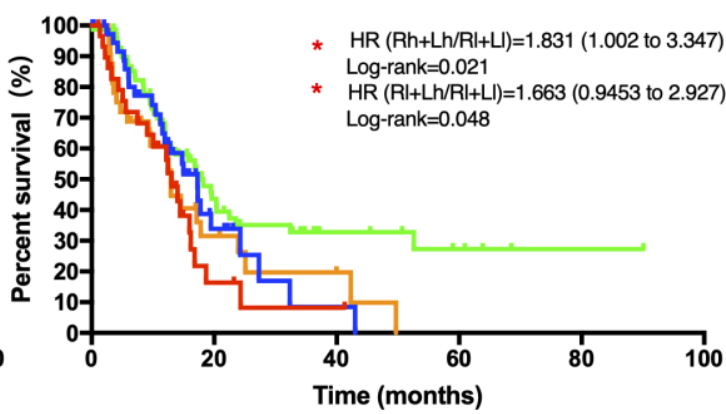

Figure 6. The clinical application value of RUNX2 and LAMC2 expression. (A) Expression levels of RUNX2 and LAMC2 in pancreatic cancer (PC) patients of different clinical stages. (B) Expression levels of RUNX2 and LAMC2 in different sex groups of PC patients. (C) Expression levels of RUNX2 and LAMC2 in different age groups of PC patients. (D) Expression levels of RUNX2 and LAMC2 in different tumor subtypes of PC patients. (E) Expression levels of RUNX2 and LAMC2in different tumor status groups of PC patients. (F) Survival analysis of combined RUNX2 and LAMC2 in TCGA PAAD cohort. 
analysis of RUNX2 and LAMC2 (Figure 6F and Supplementary Tables 1,2).

\section{LAMC2 and RUNX2 regulate $\mathrm{PC}$ cell growth and migration}

For this, we knocked down LAMC2 or RUNX2 using shRNAs in ASPC-1 cells (Figure 7A, 7B) and assessed the subsequent cell growth (Figure 7C, 7D) and migration (Figure 7E). We found that knockdown of LAMC2 or RUNX2 both inhibited PC cell growth and migration significantly. Then, we measured changes of PI3K/AKT and MAPK pathways (Figure 8A, 8B) since their vital roles in PC progression (Figure 2C, 2D). Knockdown of RUNX2 significantly inhibited phosphorylation of AKT, which implied an important
A

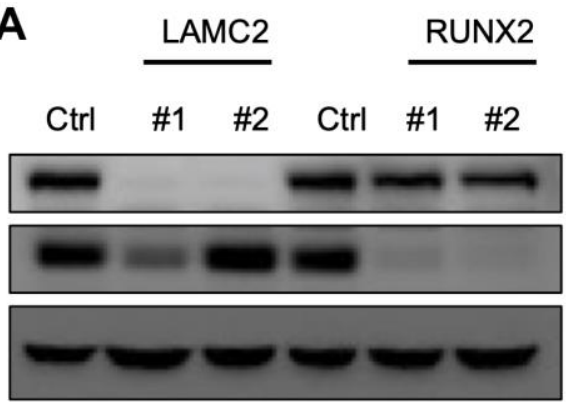

LAMC2

RUNX2

$\beta$-Actin

\section{B}

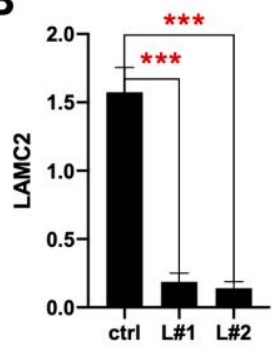

E

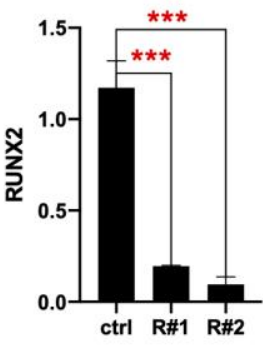

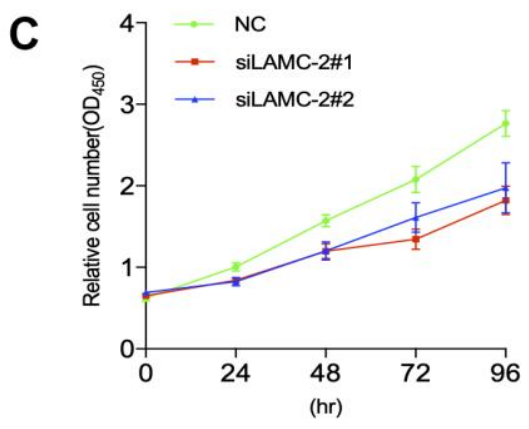

D

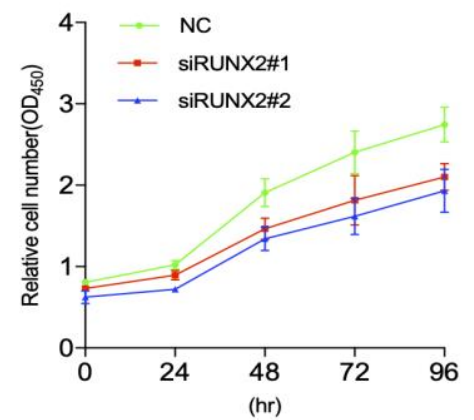

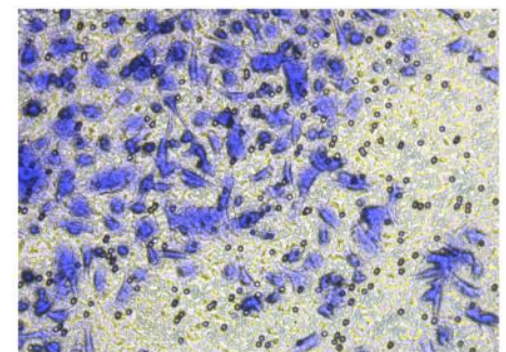

shNC

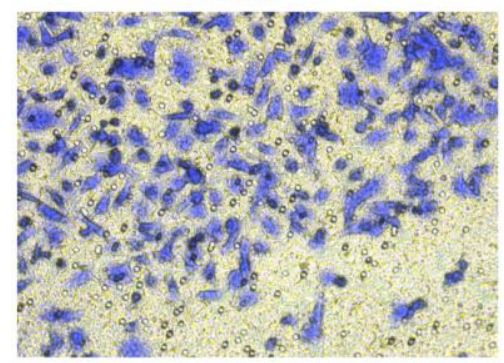

shNC

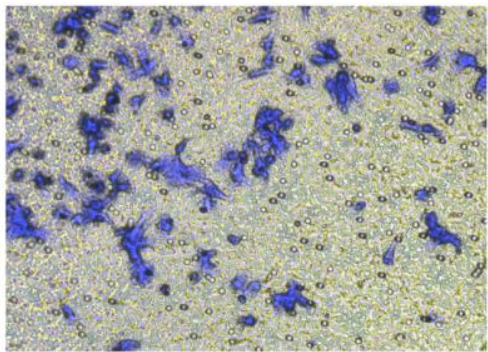

shLAMC2\#1

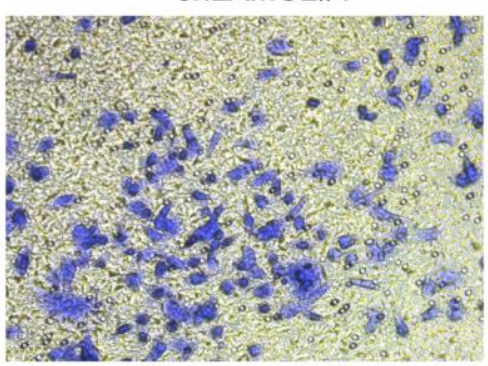

shRUNX2\#1

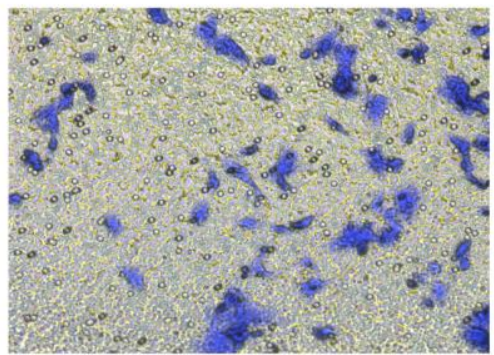

shLAMC2\#2

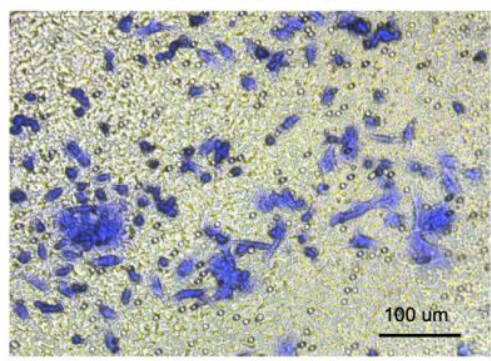

shRUNX2\#2

Figure 7. LAMC2 and RUNX2 participate in PC cell growth and migration. (A, B) ASPC-1 cells were transfected with control shRNA (NC) or two shRNAs of different sequences targeting LAMC2 or RUNX2 for $48 \mathrm{~h}$; cell samples were collected and subjected to western blot analysis. (C, D) Cell growth curves of control, LAMC2- and RUNX2-depleted cells were measured by RTCA (Real Time Cellular Analysis). (E) Cell migration of control, LAMC2- and RUNX2-depleted cells were plotted by transwell assay. 
role of RUNX2/AKT in the progression of PC. Knockout LAMC2 had no significant effect on the PI3K/AKT and MAPK pathways, but significantly reduced the expression of vimentin which is upregulated during epithelial-mesenchymal transition (Supplementary Figure 3).

\section{DISCUSSION}

Recently, the increasing morbidity and mortality of PC has made it a serious challenge for human health. However, benefiting from the development of bioinformatics technology, it is much easier for us to identify the general genetic alterations in diseases now, which may shed light on the determination of hub targets for clinical utility. Studies of miRNA biology have expanded considerably since first discovery, and have been considered as attractive targets because of their crucial roles in modulation of gene expression under healthy, inflamed and carcinogenic pathological state. Therefore, analysing gene expression changes in combination with miRNAs is vital for identifying general diagnostic and prognostic biomarkers in cancer.
A

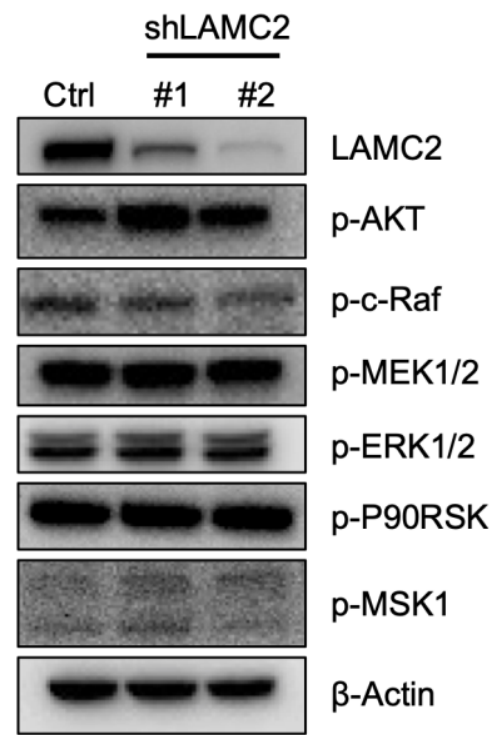

B

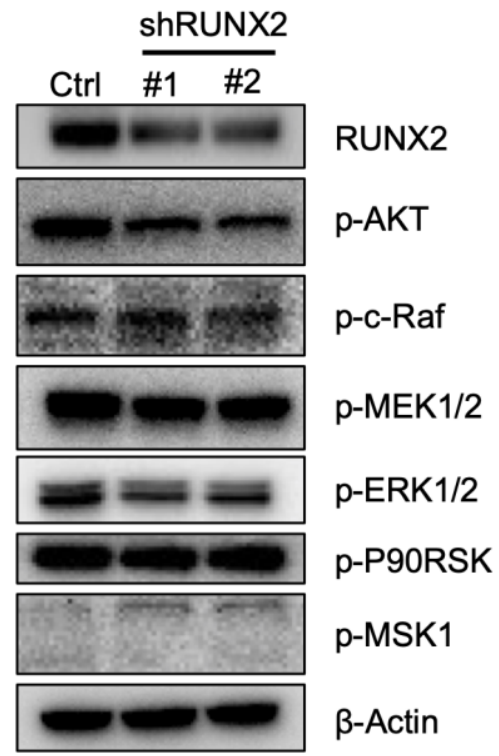

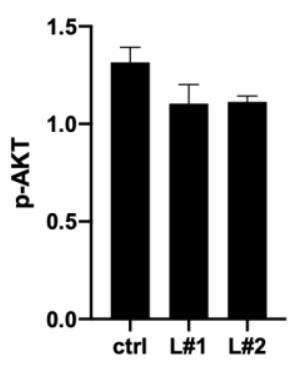
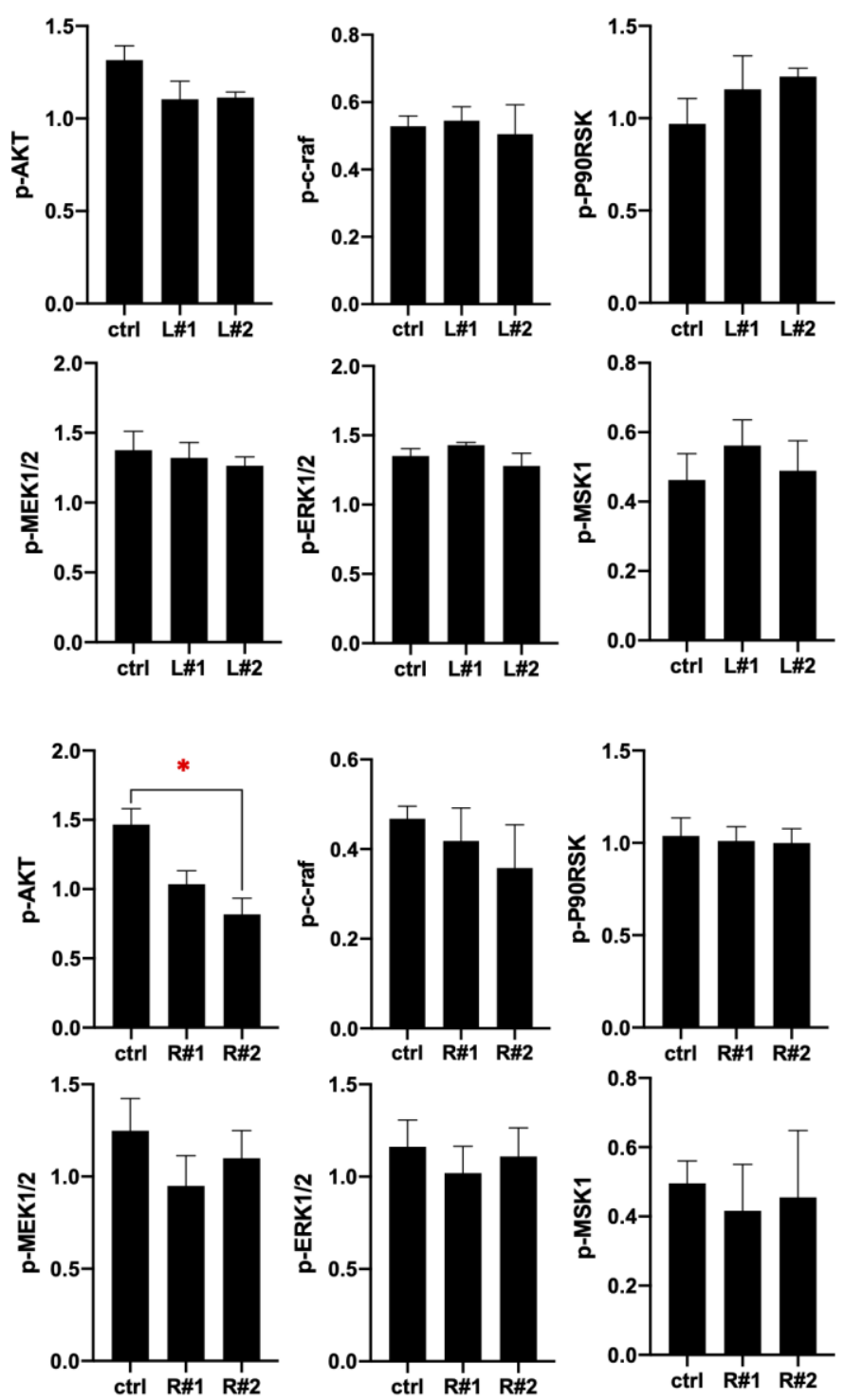

Figure 8. LAMC2 and RUNX2 regulate PC cell growth and migration through PI3K/AKT and MAPK pathway. (A, B) ASPC-1 cells were stably transfected with control shRNA (NC) or two shRNAs of different sequences targeting LAMC2 or RUNX2; cell samples were collected and subjected to Western blot analysis of p-AKT, p-c-Raf, p-MEK1/2, p-ERK1/2, p-P90RSK and p-MSK1. Actin was used as a loading control. The data expressed in right graphs represent the mean \pm SEM $(* p<0.05)$. 
In the present study, we initially found 114 tDEGs and 114 tDEMs from GEO datasets respectively. By GO function and KEGG analysis, we gained a deep understanding of these genes attached to the initiation and progression of PC. Further, to study the general profiles of molecular alterations in $\mathrm{PC}$, we then identified 3 key genes and 35 important miRNAs by aligning tDEMTGs with tDEGs. We investigated diagnostic and prognostic value of them by ROC and survival tests. And we also confirmed protein expression through IHC and WB assays. Based on the data from this study, we suggested that LAMC2 and RUNX2 could serve as potential biomarkers for the clinical use of PC in the future.

MiRNAs are a class of non-coding RNAs which can bond to 3'-untranslated region (3'-UTR) of targeted mRNA to regulate their protein expression levels [23]. Multiple studies have demonstrated that miRNAs participate in the management of all cancer hallmarks. It is generally believed that miRNAs could be important molecular tools for non-invasive diagnosis and prognosis of cancer. Therefore, it is of great importance to identify most commonly suitable DEGs which could be used as treatment targets or diagnostic markers by discussing the interaction of miRNAs and mRNAs. In this study, we finally obtained 8 key miRNAs from candidate miRNAs by further screening through ROC and survival analysis, which enriched miRNA profiles of PC and may help to highlight the diagnostic and therapeutic potential of the miRNAs cluster in PC (Figure 9).

RUNX2 belongs to RUNX family and is known as one of the main determinants of osteoblast differentiation and bone formation. Recent studies found RUNX2 was overexpressed in several tumor tissues and may play a vital role in tumor initiation, progression, invasion and metastasis [24-28]. This study demonstrated that although overexpression of RUNX2 was related to malignant behaviours, it didn't significantly affect the survival time of the PC patients which might be a bit inconsistent with previous research. Intriguingly, we found that RUNX2 presented a high diagnostic capability in PC. Histology is an invasive test that is inappropriate for the early diagnosis of PDAC, in terms of safety. Therefore, blood or body fluid tests can provide a good alternative method to assist clinicians with diagnosis. In contrast to the high expression of RUNX2 in pancreatic tissue, it was down-regulated in blood datasets, and its expression in peripheral blood mononuclear cell (PBMC, GSE49641) was significantly lower in PC group than in healthy control samples. Saliva samples (GSE14245) showed a similar result which suggests its applicability as a non-invasive diagnostic biomarker. However, there was no significant change in peripheral blood (GSE74629 and GSE15932). In addition, RUNX2 has been reported that could regulate many carcinogenesis genes and molecules in PC, including VEGF (vascular endothelial growth factor), matrix metalloproteinases and survivin [29]. Combined with the results of transcription factor prediction, we believed that the role of RUNX2 in PC progression might concentrate more on regulating the target genes, such as LAMC2.

LAMC2, a member of laminins family, is the main structural component of basement membranes and participates in a wide variety of biological processes including cell adhesion, differentiation, migration and metastasis $[30,31]$. In this study, the result of the enrichment analysis indicated that LAMC2 was closely related with the process of cell adhesion, extracellular matrix organization/disassembly and positive regulation of cell proliferation. We then found that the expression of LAMC2 was elevated in PC tumor tissues through the GEO database, IHC and WB analysis. Additionally, for PC patients with high LAMC2 expression, the prognosis was poor. According to these data, we hypothesized that the expression of LAMC2 may lead to an increased risk of tumor recurrence. Pancreatic ductal adenocarcinoma (PDAC) accounts for most

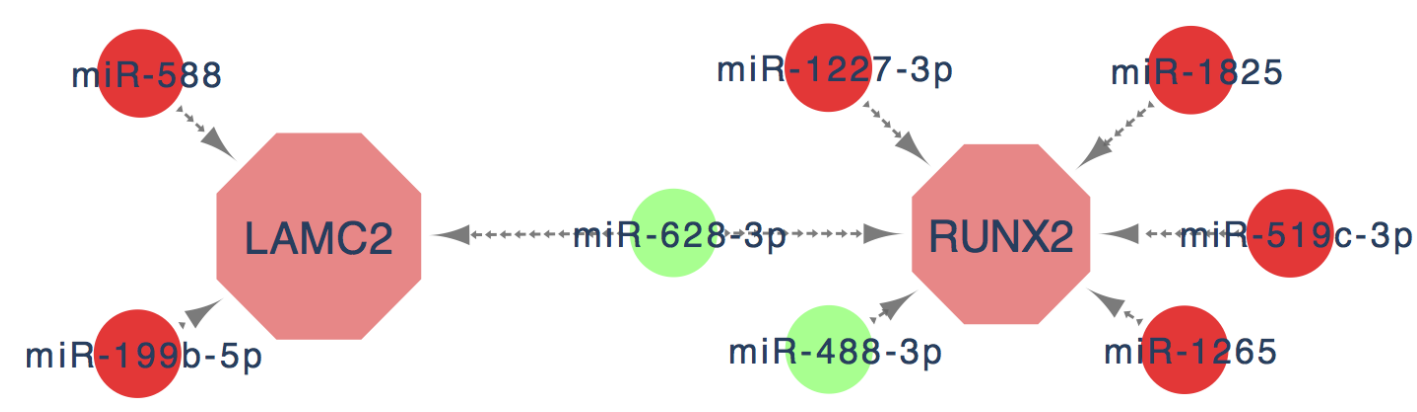

Figure 9. The key genes and key miRNA interaction network. Red nodes denote up-regulated key genes or miRNAs, while green nodes denote down-regulated key genes or miRNAs. The lines represent an interaction relationship between the nodes. 
human PC cases (more than 95\%) [32], we found that the expression of LAMC2 is mainly up-regulated in PDAC, which indicates that it is not only suitable for most PC patients, but may also help to identify the subtypes of PC. Because carbohydrate antigen 19-9 (CA19-9) which acts as the most commonly used PC marker is not accurate for the diagnosis of PDAC [33], combining with the good discriminating ability that LAMC2 showed in the AUC curve, we suggest that it may be a potential indicator in the auxiliary diagnosis of PC.

The PI3K/AKT and MAPK pathways are both important intracellular signal transduction cascades which could regulate cell growth and proliferation, survival and apoptosis, and mobility and invasion [34-36]. In tumorigenesis, dysregulation of PI3K/ AKT and/or MAPK pathways occurs in almost onethird of human cancers, especially PC [36-42]. RAS is a common signaling component of both the MAPK and PI3K/AKT signaling pathways. And PDAC is the predominant form of PC. Recently, it has been reported that KRAS is the most frequently mutated gene $(\sim 95 \%)$ in PDAC $[43,44]$. Consistent with previous research, in this study, there is a significant recruitment outcome for PI3K/AKT (for tDEGs) and MAPK signaling pathways (for tDEmiRs). Substantial efforts have been invested in developing the inhibitors of the PI3K/AKT and MAPK pathways; however, ascribed to complex crosstalk, clinical benefits are largely limited [43]. The interaction of RUNX2 and LAMC2 with the PI3K/AKT and MAPK signaling pathways can act as a driving force in controlling tumor progression [45, 46]. As a downstream molecule of the PI3K/AKT and MAPK pathway, RUNX2 can regulate metastatic properties of human prostate cancer and breast cancer cells [46-50]. However, RUNX2 also activates the PI3K/AKT pathway by regulating its components [51, 52]. Previous research has identified that the expression of LAMC2 depends largely on PI3K/AKT and MAPK pathways, and its overexpression also has a feedback regulation on these two pathways [53]. In non-small cell lung cancer cells, the inhibition of Akt upregulates LAMC2 expression, while high LAMC2 suppresses Akt signaling [53, 54]. Degen et al. found that MAPK/ERK pathway hyperactivation as the driver of LAMC $\square 2$ overexpression by neoplastic cells, correlated with increased phosphorylation and activation of the translation factors S6 and eIF4B [55]. And overexpression of LAMC2 increased the protein expression of p38 but not ERK1/2, JNK or ERK5 [56]. In this study, we found that knockdown of LAMC2 or RUNX2 inhibited PI3K/AKT and MAPK/ERK pathways to a more or less extent, especially RUNX2. These may suggest directions for the discovery of the agents' combination that target $\mathrm{PI} 3 \mathrm{~K} / \mathrm{AKT}$ and MAPK/ERK pathways in PC.

In summary, we confirmed that RUNX2 and LAMC2 are key genes that facilitate the progression of PC through bioinformatics and experimental analysis. Eight key miRNAs (miR-588, miR-199b, miR-1227, miR628, miR-488, miR-1825, miR-519 and miR1265) which participated in the regulation of key genes expression could enrich the specific miRNA profiles of PC. These findings provide a new perspective on the underlying molecular mechanism of PC, suggesting that LAMC2 and RUNX2 may be valuable biomarkers and therapeutic targets for PC patients and may also offer powerful evidence and clues for the future genomic individualized treatment of PC.

\section{AUTHOR CONTRIBUTIONS}

Guihua Jin have drafted the work and performed experiments. Qingqing Ruan and Fugen Shangguan have collected data and substantively revised it. Linhua Lan have designed of this work. All authors read and approved the final manuscript.

\section{ACKNOWLEDGMENTS}

We wish to thank the GEO database, TCGA group, miRbase database, miRWalk database, DIANA TOOLs, HPA, GEPIA, Kaplan Meier plotter and TBtools for providing relevant data and tools that is publicly accessible. We are thankful to Dr. Abdullahi Mohamed Said (Wenzhou Medical University, Wenzhou, China) for his linguistic assistance during the preparation of this manuscript.

\section{CONFLICTS OF INTEREST}

The authors declare that they have no conflicts of interest.

\section{FUNDING}

This work was supported by Natural Science Foundation Youth Fund Project of China (No. 81902803); the Natural Science Foundation of Zhejiang Province (No. LQ21H160043); and the Basic Scientific Research Project of Wenzhou (No. Y2020208).

\section{REFERENCES}

1. Siegel RL, Miller KD, Jemal A. Cancer statistics, 2019. CA Cancer J Clin. 2019; 69:7-34. https://doi.org/10.3322/caac.21551 PMID: $\underline{30620402}$ 
2. Almeida PP, Cardoso CP, de Freitas LM. PDAC-ANN: an artificial neural network to predict pancreatic ductal adenocarcinoma based on gene expression. BMC Cancer. 2020; 20:82.

https://doi.org/10.1186/s12885-020-6533-0

PMID:32005189

3. Yang C, Liu Z, Zeng X, Wu Q, Liao X, Wang X, Han C, Yu T, Zhu G, Qin W, Peng T. Evaluation of the diagnostic ability of laminin gene family for pancreatic ductal adenocarcinoma. Aging (Albany NY). 2019; 11:3679-703.

https://doi.org/10.18632/aging.102007 PMID:31182680

4. Long NP, Jung $\mathrm{KH}$, Anh NH, Yan $\mathrm{HH}$, Nghi TD, Park S, Yoon SJ, Min JE, Kim HM, Lim JH, Kim JM, Lim J, Lee S, et al. An Integrative Data Mining and Omics-Based Translational Model for the Identification and Validation of Oncogenic Biomarkers of Pancreatic Cancer. Cancers (Basel). 2019; 11:155.

https://doi.org/10.3390/cancers11020155

PMID:30700038

5. Janky R, Binda MM, Allemeersch J, Van den Broeck A, Govaere O, Swinnen JV, Roskams T, Aerts S, Topal B. Prognostic relevance of molecular subtypes and master regulators in pancreatic ductal adenocarcinoma. BMC Cancer. 2016; 16:632. https://doi.org/10.1186/s12885-016-2540-6 PMID:27520560

6. Badea L, Herlea V, Dima SO, Dumitrascu T, Popescu I. Combined gene expression analysis of whole-tissue and microdissected pancreatic ductal adenocarcinoma identifies genes specifically overexpressed in tumor epithelia. Hepatogastroenterology. 2008; 55:2016-27. PMID:19260470

7. Donahue TR, Tran LM, Hill R, Li Y, Kovochich A, Calvopina JH, Patel SG, Wu N, Hindoyan A, Farrell JJ, Li $\mathrm{X}$, Dawson DW, Wu $\mathrm{H}$. Integrative survival-based molecular profiling of human pancreatic cancer. Clin Cancer Res. 2012; 18:1352-63.

https://doi.org/10.1158/1078-0432.CCR-11-1539

PMID:22261810

8. Bauer AS, Keller A, Costello E, Greenhalf W, Bier M, Borries A, Beier M, Neoptolemos J, Büchler M, Werner J, Giese N, Hoheisel JD. Diagnosis of pancreatic ductal adenocarcinoma and chronic pancreatitis by measurement of microRNA abundance in blood and tissue. PLoS One. 2012; 7:e34151.

https://doi.org/10.1371/journal.pone.0034151 PMID:22511932

9. Chen C, Xia R, Chen H, He Y. TBtools, a Toolkit for Biologists integrating various HTS-data handling tools with a user-friendly interface. bioRxiv. 2018; 289660.

https://doi.org/10.1101/289660
10. Maragkakis $M$, Vergoulis $T$, Alexiou $P$, Reczko $M$, Plomaritou K, Gousis M, Kourtis K, Koziris N, Dalamagas T, Hatzigeorgiou AG. DIANA-microT Web server upgrade supports Fly and Worm miRNA target prediction and bibliographic miRNA to disease association. Nucleic Acids Res. 2011; 39:W145-48. https://doi.org/10.1093/nar/gkr294 PMID:21551220

11. Chou $\mathrm{CH}$, Shrestha $\mathrm{S}$, Yang CD, Chang NW, Lin YL, Liao KW, Huang WC, Sun TH, Tu SJ, Lee WH, Chiew MY, Tai CS, Wei TY, et al. miRTarBase update 2018: a resource for experimentally validated microRNA-target interactions. Nucleic Acids Res. 2018; 46:D296-302. https://doi.org/10.1093/nar/gkx1067 PMID:29126174

12. Sticht C, De La Torre C, Parveen A, Gretz N. miRWalk: An online resource for prediction of microRNA binding sites. PLoS One. 2018; 13:e0206239.

https://doi.org/10.1371/journal.pone.0206239 PMID:30335862

13. Jiao X, Sherman BT, Huang da W, Stephens R, Baseler MW, Lane HC, Lempicki RA. DAVID-WS: a stateful web service to facilitate gene/protein list analysis. Bioinformatics. 2012; 28:1805-06.

https://doi.org/10.1093/bioinformatics/bts251 PMID:22543366

14. Nagy Á, Lánczky A, Menyhárt O, Győrffy B. Validation of miRNA prognostic power in hepatocellular carcinoma using expression data of independent datasets. Sci Rep. 2018; 8:9227.

https://doi.org/10.1038/s41598-018-27521-y PMID:29907753

15. Tang Z, Kang B, Li C, Chen T, Zhang Z. GEPIA2: an enhanced web server for large-scale expression profiling and interactive analysis. Nucleic Acids Res. 2019; 47:W556-60.

https://doi.org/10.1093/nar/gkz430

PMID:31114875

16. Irigoyen A, Jimenez-Luna $C$, Benavides $M$, Caba $O$, Gallego J, Ortuño FM, Guillen-Ponce C, Rojas I, Aranda E, Torres C, Prados J. Integrative multi-platform metaanalysis of gene expression profiles in pancreatic ductal adenocarcinoma patients for identifying novel diagnostic biomarkers. PLoS One. 2018; 13:e0194844.

https://doi.org/10.1371/journal.pone.0194844 PMID:29617451

17. Caba O, Prados J, Ortiz R, Jiménez-Luna C, Melguizo C, Alvarez PJ, Delgado JR, Irigoyen A, Rojas I, PérezFlorido J, Torres C, Perales S, Linares A, Aránega A. Transcriptional profiling of peripheral blood in pancreatic adenocarcinoma patients identifies diagnostic biomarkers. Dig Dis Sci. 2014; 59:2714-20.

https://doi.org/10.1007/s10620-014-3291-3 PMID:25069573 
18. Shi M, Chen MS, Sekar K, Tan CK, Ooi LL, Hui KM. A blood-based three-gene signature for the non-invasive detection of early human hepatocellular carcinoma. Eur J Cancer. 2014; 50:928-36.

https://doi.org/10.1016/j.ejca.2013.11.026

PMID:24332572

19. Zhang L, Farrell JJ, Zhou H, Elashoff D, Akin D, Park NH, Chia D, Wong DT. Salivary transcriptomic biomarkers for detection of resectable pancreatic cancer. Gastroenterology. 2010; 138:949-57.e1. https://doi.org/10.1053/j.gastro.2009.11.010 PMID:19931263

20. Uhlén $M$, Björling $E$, Agaton $C$, Szigyarto CA, Amini B, Andersen $E$, Andersson AC, Angelidou $P$, Asplund A, Asplund C, Berglund L, Bergström K, Brumer H, et al. A human protein atlas for normal and cancer tissues based on antibody proteomics. Mol Cell Proteomics. 2005; 4:1920-32.

https://doi.org/10.1074/mcp.M500279-MCP200 PMID:16127175

21. Lan L, Wei W, Zheng Y, Niu L, Chen X, Huang D, Gao Y, Mo S, Lu J, Guo M, Liu Y, Lu B. Deferoxamine suppresses esophageal squamous cell carcinoma cell growth via ERK1/2 mediated mitochondrial dysfunction. Cancer Lett. 2018; 432:132-43.

https://doi.org/10.1016/i.canlet.2018.06.012

PMID:29906487

22. Robin X, Turck N, Hainard A, Tiberti N, Lisacek F, Sanchez JC, Müller M. pROC: an open-source package for R and S+ to analyze and compare ROC curves. BMC Bioinformatics. 2011; 12:77.

https://doi.org/10.1186/1471-2105-12-77

PMID:21414208

23. Rupaimoole R, Slack FJ. MicroRNA therapeutics: towards a new era for the management of cancer and other diseases. Nat Rev Drug Discov. 2017; 16:203-22. https://doi.org/10.1038/nrd.2016.246 PMID:28209991

24. Li H, Zhou RJ, Zhang GQ, Xu JP. Clinical significance of RUNX2 expression in patients with nonsmall cell lung cancer: a 5-year follow-up study. Tumour Biol. 2013; 34:1807-12.

https://doi.org/10.1007/s13277-013-0720-4 PMID:23471668

25. Wang ZQ, Keita M, Bachvarova M, Gobeil S, Morin C, Plante M, Gregoire J, Renaud MC, Sebastianelli A, Trinh $X B$, Bachvarov D. Inhibition of RUNX2 transcriptional activity blocks the proliferation, migration and invasion of epithelial ovarian carcinoma cells. PLoS One. 2013; 8:e74384 https://doi.org/10.1371/journal.pone.0074384 PMID:24124450
26. Zhou S, Zhang S, Wang L, Huang S, Yuan Y, Yang J, Wang $\mathrm{H}$, Li X, Wang P, Zhou L, Yang J, Xu Y, Gao H, et al. BET protein inhibitor JQ1 downregulates chromatin accessibility and suppresses metastasis of gastric cancer via inactivating RUNX2/NID1 signaling. Oncogenesis. 2020; 9:33.

https://doi.org/10.1038/s41389-020-0218-z PMID:32157097

27. Fritz AJ, Hong D, Boyd J, Kost J, Finstaad KH, Fitzgerald MP, Hanna S, Abuarqoub AH, Malik M, Bushweller J, Tye C, Ghule P, Gordon J, et al. RUNX1 and RUNX2 transcription factors function in opposing roles to regulate breast cancer stem cells. J Cell Physiol. 2020; 235:7261-72.

https://doi.org/10.1002/jcp.29625 PMID:32180230

28. Boregowda RK, Olabisi OO, Abushahba W, Jeong BS, Haenssen KK, Chen W, Chekmareva M, Lasfar A, Foran DJ, Goydos JS, Cohen-Solal KA. RUNX2 is overexpressed in melanoma cells and mediates their migration and invasion. Cancer Lett. 2014; 348:61-70.

https://doi.org/10.1016/i.canlet.2014.03.011 PMID:24657655

29. Akech J, Wixted JJ, Bedard K, van der Deen M, Hussain $S$, Guise TA, van Wijnen AJ, Stein JL, Languino LR, Altieri DC, Pratap J, Keller E, Stein GS, Lian JB. Runx2 association with progression of prostate cancer in patients: mechanisms mediating bone osteolysis and osteoblastic metastatic lesions. Oncogene. 2010; 29:811-21.

https://doi.org/10.1038/onc.2009.389 PMID:19915614

30. Garg M, Braunstein G, Koeffler HP. LAMC2 as a therapeutic target for cancers. Expert Opin Ther Targets. 2014; 18:979-82. https://doi.org/10.1517/14728222.2014.934814 PMID:24976367

31. Kosanam H, Prassas I, Chrystoja CC, Soleas I, Chan A, Dimitromanolakis A, Blasutig IM, Rückert $F$, Gruetzmann R, Pilarsky C, Maekawa M, Brand R, Diamandis EP. Laminin, gamma 2 (LAMC2): a promising new putative pancreatic cancer biomarker identified by proteomic analysis of pancreatic adenocarcinoma tissues. Mol Cell Proteomics. 2013; 12:2820-32.

https://doi.org/10.1074/mcp.M112.023507 PMID:23798558

32. Tanaka S. Molecular Pathogenesis and Targeted Therapy of Pancreatic Cancer. Ann Surg Oncol. 2016 (Suppl 2); 23:S197-205. https://doi.org/10.1245/s10434-015-4463-x PMID:25749932

33. Molina V, Visa L, Conill C, Navarro S, Escudero JM, Auge JM, Filella $X$, Lopez-Boado MA, Ferrer J, Fernandez-Cruz L, Molina R. CA 19-9 in pancreatic cancer: retrospective evaluation of patients with 
suspicion of pancreatic cancer. Tumour Biol. 2012; 33:799-807.

https://doi.org/10.1007/s13277-011-0297-8

PMID:22203495

34. Manning BD, Toker A. AKT/PKB Signaling: Navigating the Network. Cell. 2017; 169:381-405.

https://doi.org/10.1016/i.cell.2017.04.001 PMID:28431241

35. McCubrey JA, Steelman LS, Chappell WH, Abrams SL, Wong EW, Chang F, Lehmann B, Terrian DM, Milella M, Tafuri A, Stivala F, Libra M, Basecke J, et al. Roles of the Raf/MEK/ERK pathway in cell growth, malignant transformation and drug resistance. Biochim Biophys Acta. 2007; 1773:1263-84.

https://doi.org/10.1016/i.bbamcr.2006.10.001 PMID:17126425

36. Cao Z, Liao Q, Su M, Huang K, Jin J, Cao D. AKT and ERK dual inhibitors: The way forward? Cancer Lett. 2019; 459:30-40.

https://doi.org/10.1016/i.canlet.2019.05.025 PMID:31128213

37. Jokinen E, Koivunen JP. MEK and PI3K inhibition in solid tumors: rationale and evidence to date. Ther Adv Med Oncol. 2015; 7:170-80.

https://doi.org/10.1177/1758834015571111 PMID:26673580

38. Millis SZ, Ikeda S, Reddy S, Gatalica Z, Kurzrock R. Landscape of Phosphatidylinositol-3-Kinase Pathway Alterations Across 19784 Diverse Solid Tumors. JAMA Oncol. 2016; 2:1565-73.

https://doi.org/10.1001/jamaoncol.2016.0891 PMID:27388585

39. Mundi PS, Sachdev J, McCourt C, Kalinsky K. AKT in cancer: new molecular insights and advances in drug development. Br J Clin Pharmacol. 2016; 82:943-56. https://doi.org/10.1111/bcp.13021 PMID:27232857

40. Smalley KS. A pivotal role for ERK in the oncogenic behaviour of malignant melanoma? Int J Cancer. 2003; 104:527-32. https://doi.org/10.1002/ijc.10978 PMID:12594806

41. Zou Y, Liu FY, Wu J, Wan L, Fang SF, Zhang ZY, Luo Y, Chen $\mathrm{MH}$, Huang $\mathrm{MZ}$, He M, Huang OP. Mutational analysis of the RAS/RAF/MEK/ERK signaling pathway in 260 Han Chinese patients with cervical carcinoma. Oncol Lett. 2017; 14:2427-31. https://doi.org/10.3892/ol.2017.6435 PMID:28781678

42. Wan PT, Garnett MJ, Roe SM, Lee S, Niculescu-Duvaz D, Good VM, Jones CM, Marshall CJ, Springer CJ, Barford D, Marais R, and Cancer Genome Project. Mechanism of activation of the RAF-ERK signaling pathway by oncogenic mutations of B-RAF. Cell. 2004; 116:855-67. https://doi.org/10.1016/s0092-8674(04)00215-6 PMID:15035987

43. Biankin AV, Waddell N, Kassahn KS, Gingras MC, Muthuswamy LB, Johns AL, Miller DK, Wilson PJ, Patch AM, Wu J, Chang DK, Cowley MJ, Gardiner BB, et al, and Australian Pancreatic Cancer Genome Initiative. Pancreatic cancer genomes reveal aberrations in axon guidance pathway genes. Nature. 2012; 491:399-405. https://doi.org/10.1038/nature11547 PMID:23103869

44. Bryant KL, Mancias JD, Kimmelman AC, Der CJ. KRAS: feeding pancreatic cancer proliferation. Trends Biochem Sci. 2014; 39:91-100. https://doi.org/10.1016/i.tibs.2013.12.004 PMID:24388967

45. Cohen-Solal KA, Boregowda RK, Lasfar A. RUNX2 and the PI3K/AKT axis reciprocal activation as a driving force for tumor progression. Mol Cancer. 2015; 14:137. https://doi.org/10.1186/s12943-015-0404-3 PMID:26204939

46. Liu MZ, Zhou DC, Liu Q, Xie FL, Xiang DX, Tang GY, Luo $\mathrm{SL}$. Osteogenesis activity of isocoumarin a through the activation of the PI3K-Akt/Erk cascade-activated BMP/RUNX2 signaling pathway. Eur J Pharmacol. 2019; 858:172480.

https://doi.org/10.1016/i.ejphar.2019.172480 PMID:31228453

47. Ge C, Xiao G, Jiang D, Yang $Q$, Hatch NE, Roca H, Franceschi RT. Identification and functional characterization of ERK/MAPK phosphorylation sites in the Runx2 transcription factor. J Biol Chem. 2009; 284:32533-43.

https://doi.org/10.1074/ibc.M109.040980 PMID:19801668

48. Ge C, Zhao G, Li Y, Li H, Zhao X, Pannone G, Bufo P, Santoro A, Sanguedolce F, Tortorella S, Mattoni M, Papagerakis S, Keller ET, Franceschi RT. Role of Runx2 phosphorylation in prostate cancer and association with metastatic disease. Oncogene. 2016; 35:366-76. https://doi.org/10.1038/onc.2015.91 PMID:25867060

49. Tandon M, Chen Z, Pratap J. Role of Runx2 in crosstalk between Mek/Erk and PI3K/Akt signaling in MCF-10A cells. J Cell Biochem. 2014; 115:2208-17. https://doi.org/10.1002/jcb.24939 PMID:25147082

50. Aksamitiene E, Kiyatkin A, Kholodenko BN. Cross-talk between mitogenic Ras/MAPK and survival PI3K/Akt pathways: a fine balance. Biochem Soc Trans. 2012; 40:139-46.

https://doi.org/10.1042/BST20110609

PMID:22260680

51. Fujita $T$, Azuma $Y$, Fukuyama R, Hattori $Y$, Yoshida $C$, Koida M, Ogita K, Komori T. Runx2 induces osteoblast and chondrocyte differentiation and enhances their 
migration by coupling with PI3K-Akt signaling. J Cell Biol. 2004; 166:85-95.

https://doi.org/10.1083/jcb.200401138

PMID:15226309

52. Tandon M, Chen Z, Pratap J. Runx2 activates PI3K/Akt signaling via mTORC2 regulation in invasive breast cancer cells. Breast Cancer Res. 2014; 16:R16. https://doi.org/10.1186/bcr3611 PMID:24479521

53. Zhou YM, Yao YL, Liu W, Shen XM, Shi L, Wu L. MicroRNA-134 inhibits tumor stem cell migration and invasion in oral squamous cell carcinomas via downregulation of PI3K-Akt signaling pathway by inhibiting LAMC2 expression. Cancer Biomark. 2020; 29:51-67.

https://doi.org/10.3233/CBM-191362

PMID:32568182

54. Rao G, Pierobon M, Kim IK, Hsu WH, Deng J, Moon YW, Petricoin EF, Zhang YW, Wang Y, Giaccone G. Inhibition of AKT1 signaling promotes invasion and metastasis of non-small cell lung cancer cells with K-RAS or EGFR mutations. Sci Rep. 2017; 7:7066.

https://doi.org/10.1038/s41598-017-06128-9

PMID:28765579

55. Degen $M$, Natarajan E, Barron $P$, Widlund $H R$, Rheinwald JG. MAPK/ERK-dependent translation factor hyperactivation and dysregulated laminin $\gamma_{2}$ expression in oral dysplasia and squamous cell carcinoma. Am J Pathol. 2012; 180:2462-78.

https://doi.org/10.1016/j.ajpath.2012.02.028

PMID:22546478

56. Zhang D, Guo H, Feng W, Qiu H. LAMC2 regulated by microRNA-125a-5p accelerates the progression of ovarian cancer via activating p38 MAPK signalling. Life Sci. 2019; 232:116648.

https://doi.org/10.1016/j.Ifs.2019.116648

PMID:31301414 


\section{SUPPLEMENTARY MATERIALS}

\section{Supplementary Figures}
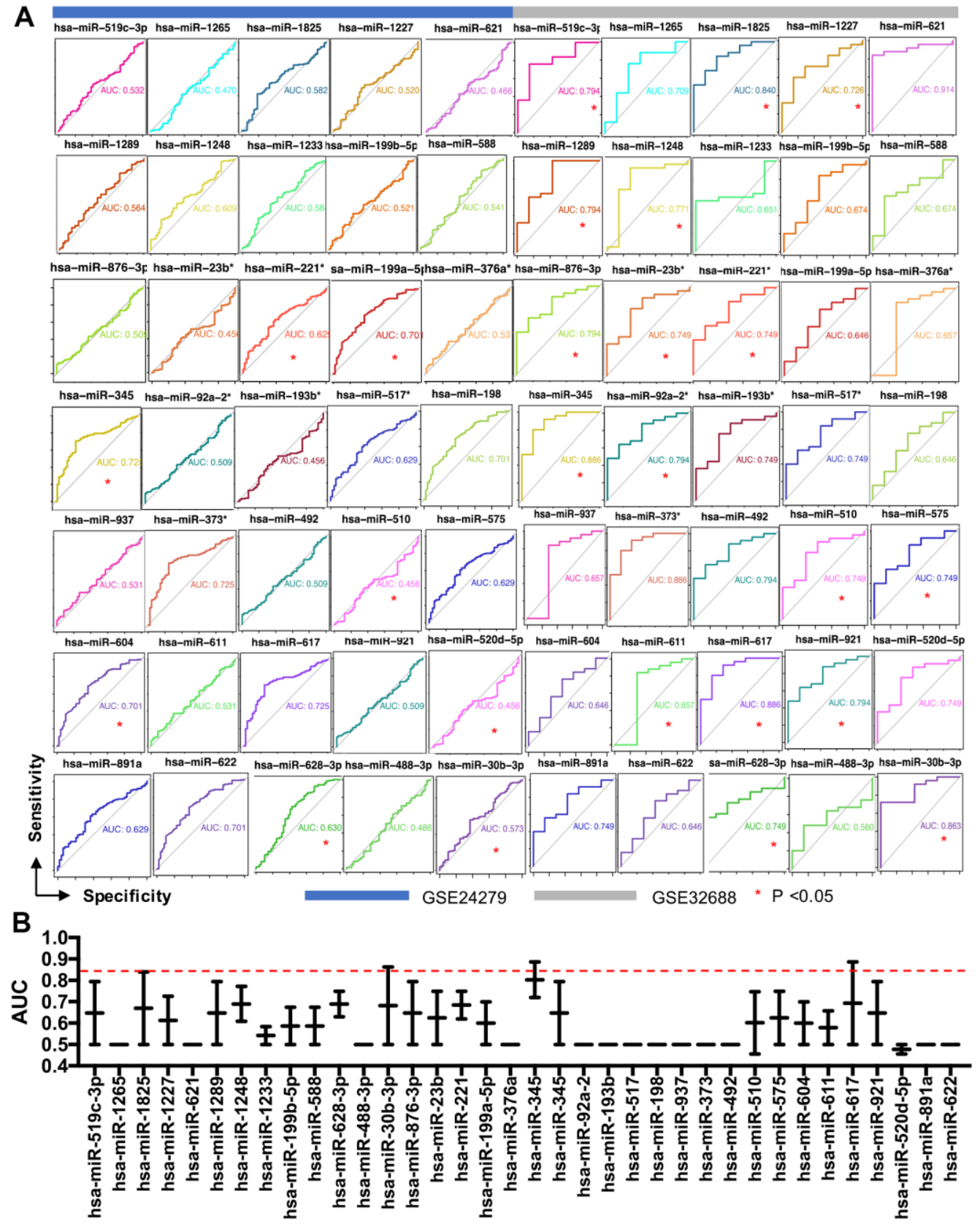

Supplementary Figure 1. ROC analysis of key genes related DEMs in GSE24279 and GSE32688. (A) ROC curve for 35 key genes related DEMs in GSE24279 and GSE32688. (B) Mean value of AUC results of 35 DEMs. 

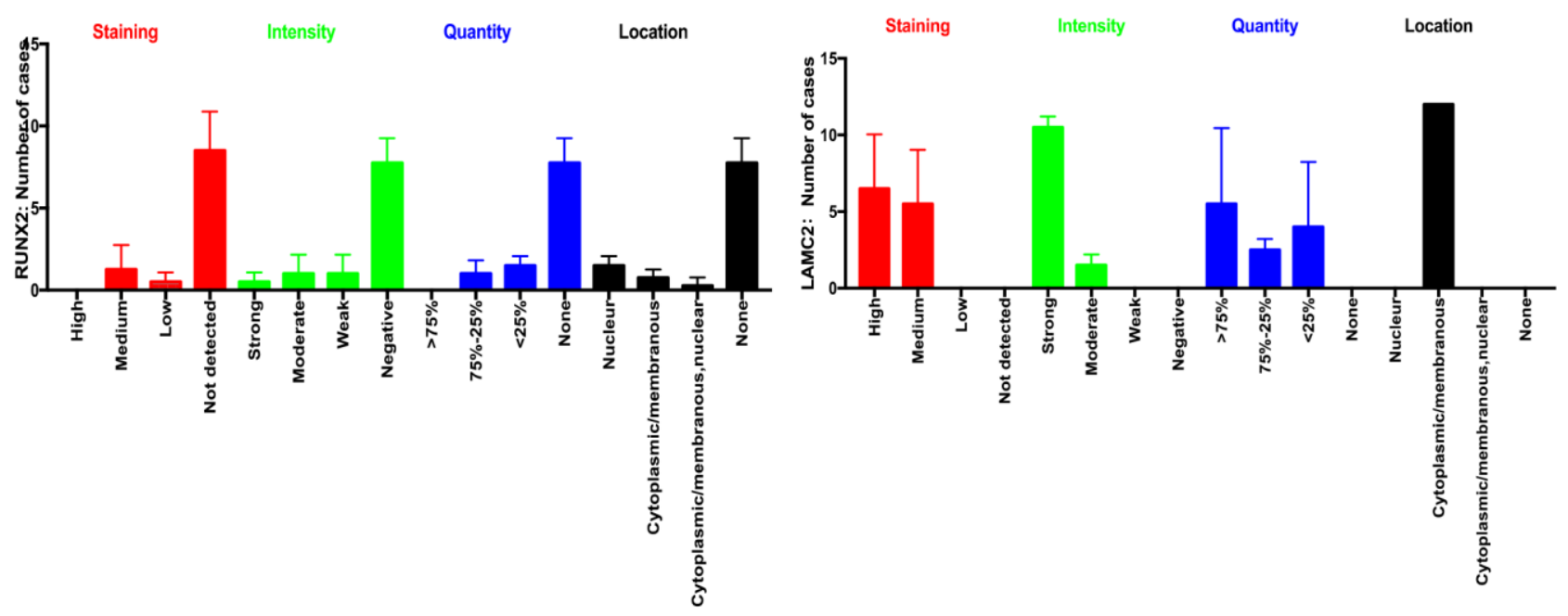

Supplementary Figure 2. The bar charts showed the summary (staining, intensity, quantity and location of RUNX2 and LAMC2) of IHC in PC patients.

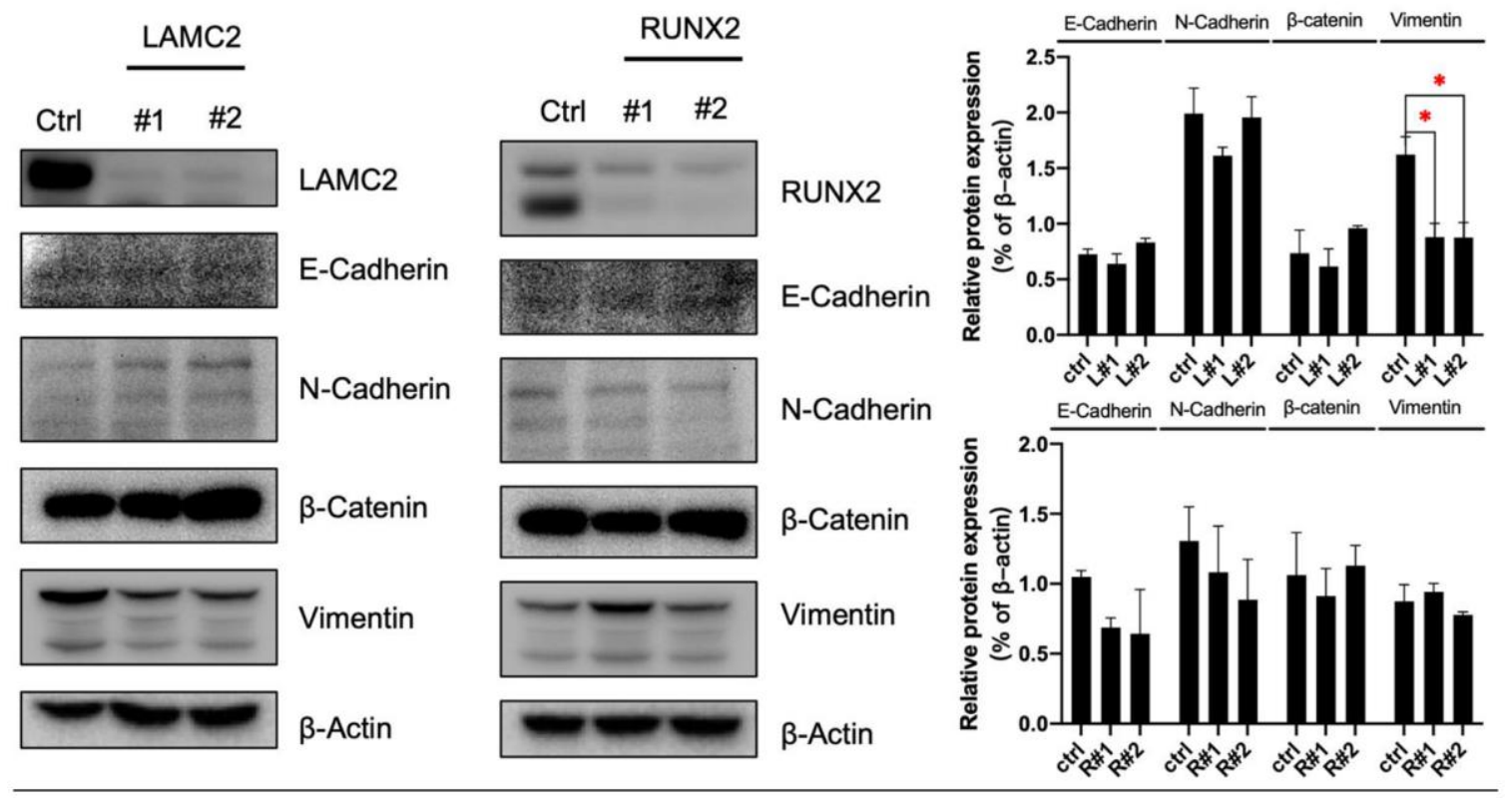

Supplementary Figure 3. ASPC-1 cells were stably transfected with control shRNA (NC) or two shRNAs of different sequences targeting LAMC2 or RUNX2; cell samples were collected and subjected to western blot analysis of E-Cadherin, $\mathrm{N}$-Cadherin, $\beta$ catenin and vimentin. 


\section{Supplementary Tables}

Supplementary Table 1. Combined OS analysis of RUNX2 and LAMC2 expression in TCGA PAAD patients.

\begin{tabular}{lccc}
\hline Variables & MST (months) & HR (95\% CI) & P value \\
\hline Low RUNX2+Low LAMC2 & 19.66 & & \\
Low RUNX2+High LAMC2 & 16.37 & $1.194(0.6816$ to 2.092$)$ & 0.5169 \\
High RUNX2+Low LAMC2 & 23.08 & $0.855(0.5011$ to 1.459$)$ & 0.5726 \\
High RUNX2+High LAMC2 & 18.67 & $1.336(0.7511$ to 2.377$)$ & 0.2879 \\
\hline
\end{tabular}

HR for the univariate Cox proportional hazards regression analysis.

Abbreviations: MST, median survival time; HR, hazard ratio; $\mathrm{Cl}$, confidence interval; PAAD, pancreatic adenocarcinoma.

Supplementary Table 2. Combined OS analysis of RUNX2 and LAMC2 expression in TCGA PAAD patients.

\begin{tabular}{lccc}
\hline Variables & MST (months) & HR (95\% CI) & P value \\
\hline Low RUNX2+Low LAMC2 & 17.92 & & \\
Low RUNX2+High LAMC2 & 12.92 & $1.663(0.9453$ to 2.927$)$ & $\mathbf{0 . 0 4 8}$ \\
High RUNX2+Low LAMC2 & 17.26 & $1.386(0.8127$ to 2.362$)$ & 0.1983 \\
High RUNX2+High LAMC2 & 13.05 & $1.831(1.002$ to 3.347) & $\mathbf{0 . 0 2 1}$ \\
\hline
\end{tabular}

HR for the univariate Cox proportional hazards regression analysis.

Abbreviations: MST, median survival time; $\mathrm{HR}$, hazard ratio; $\mathrm{Cl}$, confidence interval; PAAD, pancreatic adenocarcinoma. 